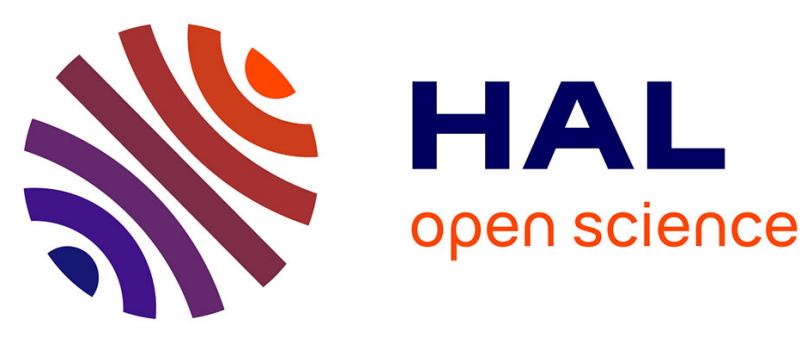

\title{
Dependence of the primary stability of cementless acetabular cup implants on their biomechanical environment
}

Maria Letizia Raffa, Vu-Hieu Nguyen, Elisabeth Tabor, Katharina Immel, Victor Housset, Charles-Henri Flouzat-Lachaniette, Guillaume Haiat

\section{To cite this version:}

Maria Letizia Raffa, Vu-Hieu Nguyen, Elisabeth Tabor, Katharina Immel, Victor Housset, et al.. Dependence of the primary stability of cementless acetabular cup implants on their biomechanical environment. Proceedings of the Institution of Mechanical Engineers, Part H: Journal of Engineering in Medicine, 2019, 233 (12), pp.1237-1249. 10.1177/0954411919879250 . hal-02284144

\section{HAL Id: hal-02284144 \\ https://hal.science/hal-02284144}

Submitted on 3 Nov 2019

HAL is a multi-disciplinary open access archive for the deposit and dissemination of scientific research documents, whether they are published or not. The documents may come from teaching and research institutions in France or abroad, or from public or private research centers.
L'archive ouverte pluridisciplinaire HAL, est destinée au dépôt et à la diffusion de documents scientifiques de niveau recherche, publiés ou non, émanant des établissements d'enseignement et de recherche français ou étrangers, des laboratoires publics ou privés. 


\section{Dependence of the primary stability of cementless acetabular cup implants on the biomechanical environment}

Maria Letizia Raffa ${ }^{\mathrm{a}}$, Vu-Hieu Nguyen ${ }^{\mathrm{b}}$, Elisabeth Tabor $^{\mathrm{a}}$, Katharina Immel ${ }^{\mathrm{a}, \mathrm{c}}$, Victor Housset ${ }^{\mathrm{d}, \mathrm{e}}$, Charles-Henri Flouzat-Lachaniette $^{\mathrm{d}, \mathrm{e}}$, Guillaume Haiat ${ }^{\mathrm{a}^{*}}$

${ }^{a}$ CNRS, Laboratoire Modélisation et Simulation Multi Echelle, MSME UMR 8208 CNRS, 61 avenue du Général de Gaulle, 94010 Créteil Cedex, France.

${ }^{b}$ Université Paris-Est, Laboratoire Modélisation et Simulation Multi Echelle, MSME UMR 8208 CNRS, 61 avenue du Général de Gaulle, 94010 Créteil Cedex, France.

${ }^{c}$ Aachen Institute for Advanced Study in Computational Engineering Science (AICES), RWTH Aachen University, Templergraben 55, 52056 Aachen, Germany

${ }^{d}$ Service de Chirurgie Orthopédique et Traumatologique du Centre Hospitalier Universitaire Henri Mondor, 51 avenue du Maréchal de Lattre de Tassigny, 94000 Créteil, France ;

${ }^{e}$ Équipe 10, Groupe 5, IMRB U955, INSERM/UPEC, 51 Avenue du Maréchal de Lattre de Tassigny, 94010 Créteil, France

Submitted to Proceedings of the Institution of Mechanical Engineers, Part H: Journal of Engineering in Medicine

*Corresponding author:

Guillaume HAÏAT

Laboratoire Modélisation et Simulation Multi Echelle, MSME UMR CNRS 8208,

61 avenue du Général de Gaulle,

94010 Créteil, France

Tel: (33) 145171441

Fax: (33) 145171433

E-mail: guillaume.haiat@u-pec.fr 


\begin{abstract}
Biomechanical phenomena occurring at the bone-implant interface during the press-fit insertion of acetabular cup (AC) implants are still poorly understood. This paper presents a nonlinear geometrical 2-D axisymmetric finite element model aiming at describing the biomechanical behavior of the AC implant as a function of the bone Young's modulus $E_{b}$, the diametric interference fit $(I F)$ and the friction coefficient $\mu$. The numerical model was compared with experimental results obtained from an in vitro test, which allows to determine a reference configuration with the parameter set: $\mu^{*}=0.3$, $E_{b} *=0.2 \mathrm{GPa}$ and $I F^{*}=1 \mathrm{~mm}$ for which the maximal contact pressure $t_{N}=10.7 \mathrm{MPa}$ was found to be localized at the peri-equatorial rim of the acetabular cavity. Parametric studies were carried out, showing that an optimal value of the pull-out force can be defined as a function of $\mu, E_{b}$, and $I F$. For the reference configuration, the optimal pull-out force is obtained for $\mu=0.6$ (respectively $E_{b}=$ $0.35 \mathrm{GPa}$ and $I F=1.4 \mathrm{~mm})$. For relatively low value of $\mu(\mu<0.2)$, the optimal value of $I F$ linearly increases as a function of $\mu$ independently of $E_{b}$, while for $\mu>0.2$, the optimal value of $I F$ has a nonlinear dependence on $\mu$ and decreases as a function of $E_{b}$. The results can be used to help surgeons determine the optimal value of $I F$ in a patient specific manner.
\end{abstract}

Keywords: bone-implant interface; acetabular cup; FE analysis; friction; interference fit; primary stability 


\section{Introduction}

Within the last thirty years, total hip arthroplasty has become a common surgical intervention (1). Nevertheless, surgical failures still occur, leading to dramatic consequences for patients and important costs for the healthcare system. Aseptic loosening is one of the most common causes of failure (2) and is often determined by the implant primary stability.

Cementless acetabular cup (AC) implant surgery has become more and more employed over the past decade (3). AC implants are inserted using the press-fit technique, which consists in obtaining the implant primary stability by slightly oversizing the AC implant compared to the host bone cavity. The $\mathrm{AC}$ implant is then inserted into the bone cavity using impacts produced by a surgical mallet. The AC implant primary stability refers to the quality of the implant mechanical fixation obtained during the surgery, which is due to residual stresses localized mostly at the AC implant rim (4-6). An adapted primary stability lies in a compromise between i) reducing the relative micromotions at the boneimplant interface (BII), which may lead to the formation of fibrous tissue in the peri-implant region (2) and ii) avoiding "too high" stresses in peri-implant bone tissue, which may lead to bone necrosis or local ischemia (7). Both phenomena may jeopardize osseointegration processes (8) and hence lead to the implant aseptic loosening. Osseointegration leads to a bonded BII after the healing process and guarantees the long-term fixation of the AC implant (9-12) into bone tissue. The AC implant stability depends on the implant properties, on the surgical protocol, as well as on the patient behavior and bone quality.

Different pull-out tests (13-15) have been carried out in vitro and ex vivo in order to assess the AC implant primary stability but they cannot be used in the operative room. Methods based on resonance frequency analyses (16-19) have been considered but remain so far difficult to be used in clinical practice. More recently, approaches based on the analysis of the time dependence of the force produced during the impact between an instrumented hammer and the ancillary (20-26) have been developed by our group. In this context, understanding the determinants of the AC implant stability could be useful to help to improve the surgical procedure.

Several experimental studies focused on the effect of bone quality $(5,27)$ on the biomechanical behavior of AC implant. Furthermore, implant manufacturers have developed new implant properties including innovative implant geometries (5, 28-30) and surface coatings (13, 15, 27, 31-33). Nevertheless, these improvements are sometimes related to empirical developments and there is a lack of systematic investigation of the multifactorial determinants of the AC implant stability. Therefore, several numerical models have been developed to assess the biomechanical behavior of the cementless $\mathrm{AC}$ implant $(6,34)$. However, the relation between bone properties and the AC implant macroscopic mechanical behavior remains poorly established. Moreover, the complex biomechanical phenomena occurring at the BII involving friction are still poorly understood (35). 
The aim of this study is to provide a better understanding of the determinants of the biomechanical primary stability of cementless AC implants. To tackle this problem, geometrically nonlinear finite element analyses of a 2-D axisymmetric model were performed to simulate the quasistatic insertion and pull-out process of the AC implant in bone. We assessed the effect of a variation of the friction coefficient $\mu$ at the BII, of the bone Young's modulus $E_{b}$ and of the interference fit $I F$ (which is defined by the difference between the implant and the bone cavity nominal diameters) within their physiological ranges on the AC implant primary stability. The choice of studying the effect of the three aforementioned parameters comes from their importance regarding the AC implant stability. The numerical results were compared with an in vitro experimental configuration.

\section{Material and methods}

\subsection{Mechanical modeling and numerical simulation}

\subsubsection{Geometry and material properties}

The proposed geometrical configuration is described schematically in Fig. 1a. The system comprised three sub-domains: the ancillary, the AC implant and the bone sample, denoted by $\Omega_{a}, \Omega_{i}$ and $\Omega_{b}$, respectively. The AC implant had an outer radius of $25.5 \mathrm{~mm}$ and its thickness varied linearly as a function of the polar angle, with values comprised between $2.9 \mathrm{~mm}$ at the cup dome and $3.7 \mathrm{~mm}$ at the cup rim, similarly as what was done in (20). This AC implant design corresponds to the acetabular cup Cerafit (Ceraver, Roissy, France). Moreover, a cylindrical ancillary was rigidly attached to the AC implant, similarly as what is done in the clinic. The ancillary's radius and height were $8.5 \mathrm{~mm}$ and $190 \mathrm{~mm}$, respectively, which corresponds approximately to materials used in the operating room. The bone sample was modeled by a cylinder with a radius equal to $50 \mathrm{~mm}$ and a height of $40 \mathrm{~mm}$, in which a hemispheric cavity was realized at the upper surface. The bone cavity diameter was varied between 48.5 and $50.9 \mathrm{~mm}$. Following $(20,32)$, the top edge of the acetabulum was smoothed in order to avoid unphysical stress concentrations by considering a radius of curvature of $2 \mathrm{~mm}$ at the top of the cavity. The aforementioned geometrical configuration corresponds to a simplistic model of the human pelvic zone, which will be discussed in Section 4.

All material properties corresponding to the subdomains $\left(\Omega_{a}, \Omega_{i}\right.$ and $\left.\Omega_{b}\right)$ described in Fig. 1a were assumed to be homogeneous, isotropic and elastic. The ancillary (subdomain $\Omega_{a}$ ) and the AC implant (subdomain $\Omega_{i}$ ) were assumed to be made of stainless steel and titanium alloy TiAl6V4, respectively. The bone subdomain $\Omega_{b}$ was assumed to be composed of trabecular bone. Table 1 presents several values of the Young modulus of trabecular bone tissue $E_{b}$ corresponding to the physiological range $(5,36)$ which were used for this study. The elastic properties of the ancillary and of the implant are also given in Table 1. 


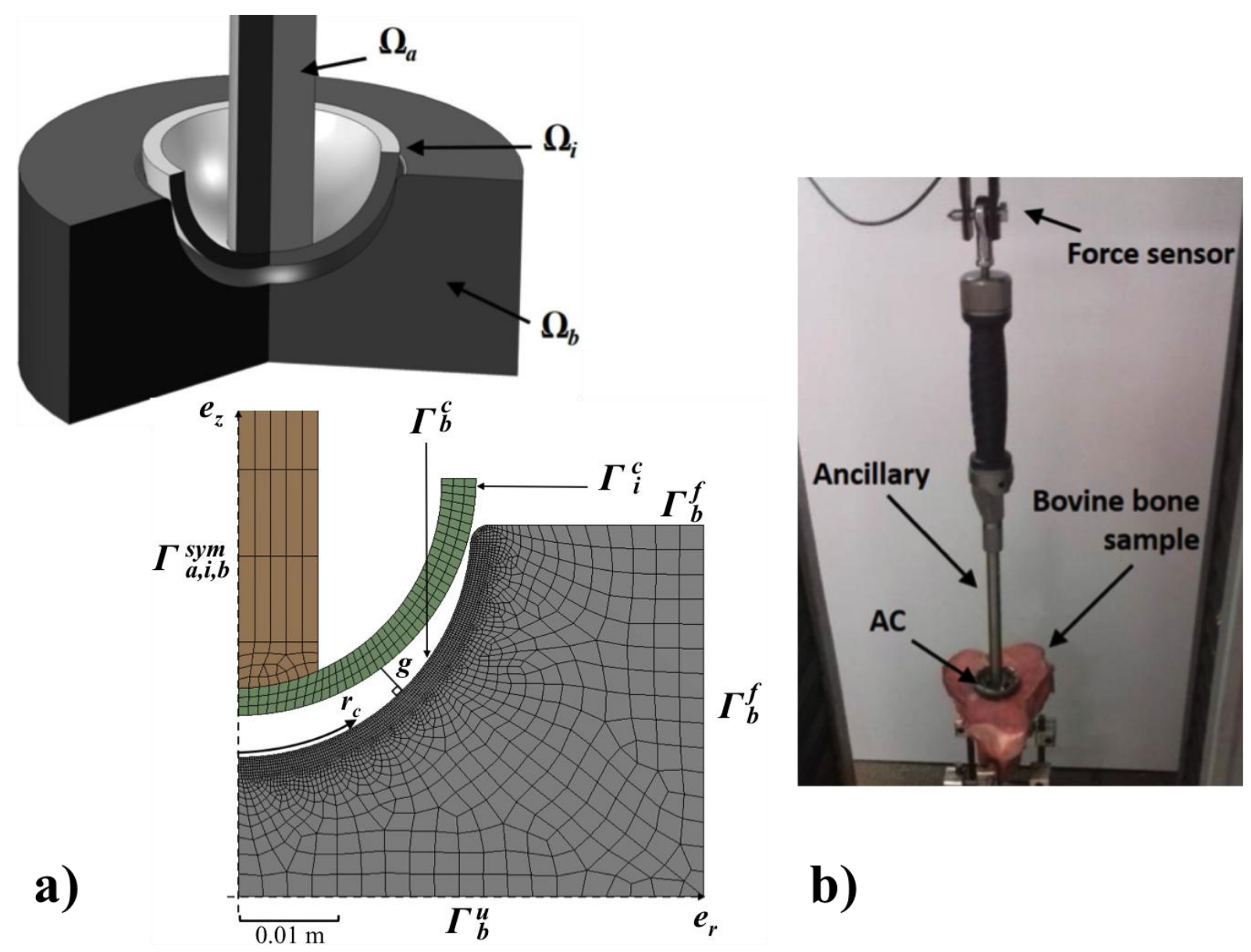

Figure 1 Mechanical and geometrical configurations for the a) in silico and b) in vitro models. a) Schematic representation of the geometrical configuration of the model including the ancillary $\left(\Omega_{a}\right)$, the implant $\left(\Omega_{i}\right)$, and the bone sample $\left(\Omega_{b}\right)$. $\Gamma_{\boldsymbol{i}}^{\boldsymbol{c}}$ and $\boldsymbol{\Gamma}_{\boldsymbol{b}}^{c}$ correspond to the contacting surfaces of the implant and the bone, respectively. $\boldsymbol{\Gamma}_{\boldsymbol{b}}^{\boldsymbol{u}}$ is the fixed boundary of the bone sample. The coordinate $r_{c}$ runs along the bone-cavity $\operatorname{arc} \boldsymbol{\Gamma}_{\boldsymbol{b}}^{c}$. The maximum value of the polar gap $g$ is obtained for $r_{c}=0 . \mathrm{b}$ ) Experimental configuration of the axial pull-out test.

Table 1 Material properties of the three subdomains considered in the model. The corresponding reference numbers are indicated.in the last line.

\begin{tabular}{c|c|c|c|c}
\hline Subdomain & Material & $\begin{array}{c}\text { Young's modulus } \\
(\mathbf{G P a})\end{array}$ & $\begin{array}{c}\text { Poisson ratio } \\
\boldsymbol{v}\end{array}$ & $\begin{array}{c}\text { Reference value } \\
\boldsymbol{E}_{\boldsymbol{b}}{ }^{*}(\mathbf{G P a})\end{array}$ \\
\hline Ancillary $\boldsymbol{\Omega}_{\boldsymbol{a}}$ & Stainless steel & 210 & 0.3 & - \\
\hline Implant $\boldsymbol{\Omega}_{\boldsymbol{i}}$ & Titanium alloy & 113 & 0.3 & - \\
\hline Bone sample $\boldsymbol{\Omega}_{\boldsymbol{b}}$ & Trabecular bone & $0.1-0.9(5,36)$ & $0.3(34)$ & $0.2(36,37)$ \\
\hline
\end{tabular}




\subsubsection{Numerical simulation method}

For the simulation of the mechanical problem corresponding to the insertion and to the pull-out of an AC implant into bone tissue, nonlinear geometrically elastic solids were considered under quasistatic loading conditions and the inertia force was neglected. The problem was expressed in terms of large displacements. Numerical analyses were carried out using Ansys ${ }^{\circledR}$ Workbench software (v. 17.0, Canonsburg, US).

An axisymmetric condition was applied on the boundaries located on the z-axis $\left(\Gamma_{a, i, b}^{s y m}\right.$ see Fig 1a). The bottom side of the bone sample $\left(\Gamma_{b}^{u}\right.$, Fig 1a) was assumed to be fixed. At the initial state $t=0$, the outer boundary of the AC implant $\Gamma_{i}^{c}$ and the internal boundary of the bone hemispherical cavity $\Gamma_{b}^{c}$ were assumed to be close but out-of-contact. Then a vertical quasi-static displacement $U_{z}(t)$ applied at the top surface of the ancillary $\Gamma_{a}^{u}$ in the axial direction $(z)$ :

$$
\boldsymbol{u}=\left[0, \quad U_{z}(t)\right]^{T} \quad \text { in } \Gamma_{a}^{u},
$$

where $U_{z}(t)$ is a piecewise three-step function defined by:

$$
\mathrm{U}_{\mathrm{z}}(t)=\left\{\begin{array}{cl}
\mathrm{d}_{0} \times\left(\mathrm{t} / \mathrm{T}_{1}\right) & \text { for } 0 \leq t<T_{1}, \\
\mathrm{~d}_{0} & \text { for } T_{1} \leq t<T_{2}, \\
\mathrm{~d}_{0} \times\left(\mathrm{T}_{2}+T_{1}-\mathrm{t}\right) / \mathrm{T}_{1} & \text { for } T_{2} \leq t<T_{2}+T_{1},
\end{array}\right.
$$

with $T_{1}=1$ (s) and $T_{2}=1.2(\mathrm{~s})$. The imposed displacement history $U_{z}(t)$ (see Fig. 2) was incremented with a step of $\Delta t=0.001 \mathrm{~s}$. Since a quasi-static regime was considered, the time step unit (s) was only indicative and did not affect the simulation results, which did not depend on the values chosen for $T_{1}$ and $T_{2}$.

As indicated in Fig. 2, an insertion or push-in phase was imposed in the time range $t \in[0,1] \mathrm{s}$, which corresponds to a linearly decreasing variation of $U_{z}(t)$ between $U_{z}(t=0)=0 \mathrm{~mm}$ (i.e., the initial value) and $U_{z}(t=1)=d_{0}$. Next, the imposed displacement remained constant in the time interval $t \in[1,1.2]$ s. Eventually, the AC implant was pulled out of bone tissue by imposing a linear increase of $U_{z}(t)$ until the implant was completely detached from bone tissue. The system was controlled in displacement. 


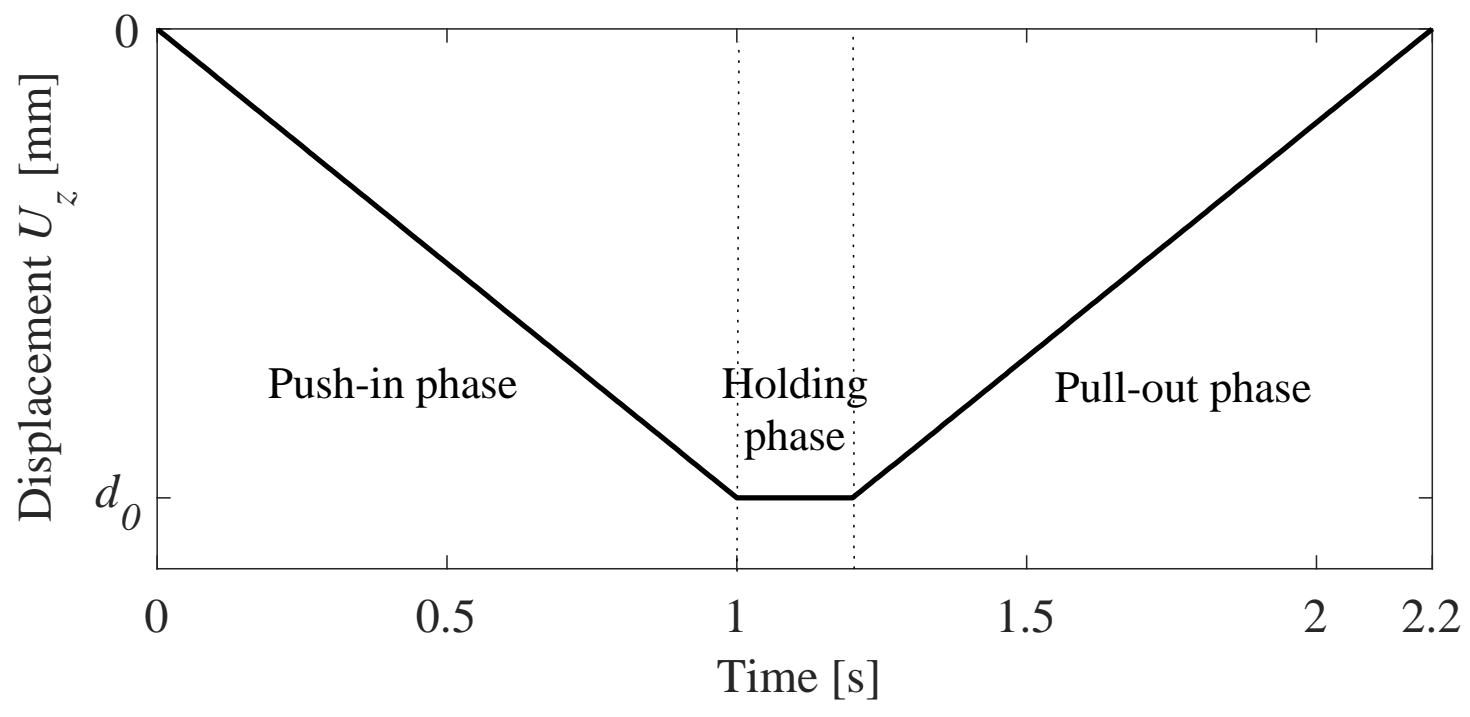

Figure 2 Variation of the displacement applied to the top of the ancillary $\boldsymbol{\Gamma}_{\boldsymbol{a}}^{\boldsymbol{u}}$ in the axial direction (z) as a function of time.

The vertical reaction force (denoted by $R_{a}(t)$ in what follows) corresponding to the vertical force applied to the ancillary was determined at each time step by integrating the vertical components of reaction stress tensor over the top surface $\Gamma_{a}^{u}$ of the ancillary. The maximum value of the imposed displacement $d_{0}$ was chosen so that $R_{a}\left(T_{1}\right)=F_{0}$, where $F_{0}$ corresponds to the press-fitting force. The pull-out force was defined as the maximum value of $R_{a}(t)$ obtained during the pull-out phase, which corresponds to an indication of the AC implant primary stability.

The contact interaction at the BII $\Gamma^{c}=\Gamma_{b}^{c} \cup \Gamma_{i}^{c}$ was governed at each time step by the unilateral contact condition together with the static Coulomb's friction law which, within a quasi-static framework (38), writes:

$$
\left\{\begin{array}{c}
\left|t_{T}\right|-\mu t_{N} \leq 0 \\
t_{N} \geq 0 \\
\mathrm{~g} \leq 0 \\
t_{N} \cdot \mathrm{g}=0
\end{array}\right.
$$

where $t_{N}=-\boldsymbol{n}^{T} \boldsymbol{\sigma} \boldsymbol{n}, t_{T}=-\boldsymbol{t}^{T} \boldsymbol{\sigma} \boldsymbol{n}$ with $\boldsymbol{n}$ and $\boldsymbol{t}$ the normal and tangential unit vectors of the bone surface; $\mu$ is the friction coefficient at the BII; $g$ is the gap function which corresponds to the normal distance between the bone's cavity surface $\Gamma_{b}^{c}$ and the implant surface $\Gamma_{i}^{c}$ (Fig 1a). The contact algorithm used to solve the Coulomb contact condition was the Augmented Lagrangian method (39). 


\subsubsection{Mesh generation and numerical convergence}

The finite element mesh used to run the numerical simulation was slightly changed depending on the geometrical parameters, but it typically contained around 2,500 quadrilateral Lagrange elements and 200 contact elements, leading to a global system with about 15,000 degrees of freedom. The quadratic interpolation function was used, which is appropriate to mesh a curved geometry (see Fig. 1a). The mesh size had to be finer around the bone cavity surface (size $0.2 \mathrm{~mm}$ ), assumed to be the "slave" surface in the computational contact conditions, compared to the "master" surface, i.e., the AC implant outer surface. Refining the mesh around the bone cavity surface was also required to avoid numerical problems in the resolution due to a non-uniform unit normal vector, both contacting surfaces being non-planar (see Fig. 1).

A convergence study was performed (data not shown) to verify that the chosen values of time increment step $(\Delta t)$ and element sizes were sufficiently small to obtain converged solutions. Numerical convergence was checked with different mesh sizes at the contacting surfaces. The contact algorithm used to solve the Coulomb contact condition was the Augmented Lagrangian method (39).

\subsubsection{Parametric study}

A parametric study was carried out in order to determine the influence of $\mu, E_{b}$ and $I F$ on the PO force, which is an indicator of the AC implant primary stability (32). A two-step methodology was adopted. First, the effects of the parameters were studied independently by varying one parameter at a time (i.e., One-Factor-At-Time OFAT design) and keeping the two other parameters equal to their reference values (see subsections 3.2.1, 3.2.2, 3.2.3) which have been identified experimentally (see section 2.2.2) and confirmed by literature. This screening allowed to identify an "optimal" stability condition (see section 3.2.4) relating $I F$ and $\mu$. Second, the effects of the coupled variations of the set $\left(I F, E_{b}, \mu\right)$ were investigated with reference to the "optimal" parameters (see details in subsection 3.2.4).

The influence of the following parameters was investigated:

- Different values of the diametric interference fit $I F$ comprised between $0.1 \mathrm{~mm}$ and $2.5 \mathrm{~mm}$ were considered according to values found in $(6,32,34,40,41)$. The reference value for the interference fit was assumed to be $I F^{*}=1 \mathrm{~mm}$, which is a standard value used in clinical practice (40).

- A wide range of values of the friction coefficient $\mu$ (between 0.1 and 1) was considered in order to simulate the physiological range of friction for various types of implant surface in contact with trabecular bone $(14,31,32,42)$ and for different clinical situations of the BII. The value $\mu=\mu^{*}=$ 0.3 was taken as the reference value following (43).

- The range of variation of the bone Young's modulus is indicated in Table 1. 


\subsection{In vitro experimental measurements}

\subsubsection{Experimental configuration}

An in vitro experimental test was carried out in order to compare with the numerical model described above. The experimental protocol was described in detail in (21) and is briefly recalled in what follows. A 50-mm nominal diameter AC implant of (Cerafit HAC, Ceraver, Roissy, France) made in titanium alloy TiAl6V4 and coated with hydroxyapatite (HA) was used. A bovine femur, obtained from the local butcher shop, was prepared by cutting the sample in a plane perpendicular to its axis in the proximal region in order to obtain a sufficient amount of trabecular bone. A hemispherical cavity was reamed at the upper surface of the bone sample using the reamer provided by the manufacturer, which was held in a clamp. Bone cavities with different diameters (within the range of $48-52 \mathrm{~mm}$ ) were successively created in the same bone sample in order to consider different values of the interference fit, similarly as what was done in (23).

The dedicated ancillary was screwed into the AC implant, which was positioned so that its longitudinal axis was aligned with the cavity symmetry axis, as shown in Fig. 1b. The AC implant was then inserted into the cavity by performing a series of impacts with a surgical mallet $(1.3 \mathrm{~kg})$ until the surgeon judged that it was completely inserted according to his proprioception, similarly as in the clinical practice.

After the AC implant insertion, a pull-out test was carried out using a mechanical testing device (MTS Systems Corporation, Eden Prairie, Minnesota, US) equipped with a force sensor (FGP instrumentation, Les Clayes-sous-Bois, France). The bone sample was rigidly fixed to the bottom part of the frame of the device through a clamp. The top part of the ancillary was attached to the top part of the rigid frame (Fig. 1b). A pull-out test was realized in displacement-controlled mode with a gradually increasing upward displacement $(0.4 \mathrm{~mm} / \mathrm{s})$. Finally, the maximum value of the force necessary to pull the AC implant out of the bone cavity was measured and is referred to as the pull-out (PO) force in what follows. The impaction and pull-out protocols were realized consecutively for each tested value of the interference fit comprised between 0 and $2 \mathrm{~mm}$.

\subsubsection{Identification of the reference parameters}

A comparison between experimental and numerical tests is a simple way of defining a reference set of parameters. The stars in Fig. 3 indicate the variation of the PO force as a function of the interference fit $I F$ obtained experimentally. The experimental results show that the PO force increases as a function of the $I F$ when $I F<1.5 \mathrm{~mm}$, reaches a maximum value of $560 \mathrm{~N}$ for $I F=1.5 \mathrm{~mm}$, and then decreases when the value of the $I F$ becomes more important $(I F=2 \mathrm{~mm})$. 
The range of variation of IF considered in Fig. 3 was obtained from $(6,32,34,40,41)$. For low values of $I F$, the variation of the pull-out force as a function of IF is shown to be linear, with a slope depending on $\left(\mu, E_{b}\right)$. The reference value of $\mu=0.3$ was chosen based on results obtained in the literature (43). Then, the value of $E_{b}$ was chosen so that the slope of the curve representing the variation of the PO force as a function of IF obtained from simulations coincides with the experimental results by using a least square fitting method. This method leads to value of $E_{b}=0.2 \mathrm{GPa}$, in good agreement with results obtained in (36-37). Numerical simulations with three different values of the press-fitting force $F_{0}(1,2.5$ and $5 \mathrm{kN})$ were then performed. These values were chosen according to experimental press-fitting force $F_{0}$ found by our group (20-26) using an instrumented hammer. Only three values were tested for the PO force because the aim was not to determine with precision the value of $F_{0}$ but to provide an order of magnitude of a reasonable value to be used in the other computations. However, it was not possible to directly compare the experimental values of $F_{0}$, which were obtained in transient regime using impact lasting several milliseconds with the numerical values of $F_{0}$, which are computed in a static mode since we do not consider transient regime (see subsection 4.3 for a discussion on this approximation). Here, we focus on the pull-out force because it is a suitable indicator of AC primary stability (32). Figure 3 also shows the variation of the PO force as a function of the interference fit $I F$ obtained numerically. When $I F$ is lower than $0.6 \mathrm{~mm}$, the PO force does not depend on $F_{0}$. The value of the press-fitting force $F_{0}=2.5 \mathrm{kN}$ leads to a relationship between PO force and $I F$ which is the most consistent with the experimental data. In this case, a maximum value of the $\mathrm{PO}$ force equal to $531.8 \mathrm{~N}$ is reached for $I F=1.4 \mathrm{~mm}$. By using the set of parameters $\left(\mu=0.3, E_{b}=0.2 \mathrm{GPa}\right.$ and $\left.F_{0}=2.5 \mathrm{kN}\right)$, the mean relative error between the five experimental results and the corresponding numerical results is equal to $13 \%$. Thus, these parameters were chosen as the reference set (noted with a star superscript in what follows).

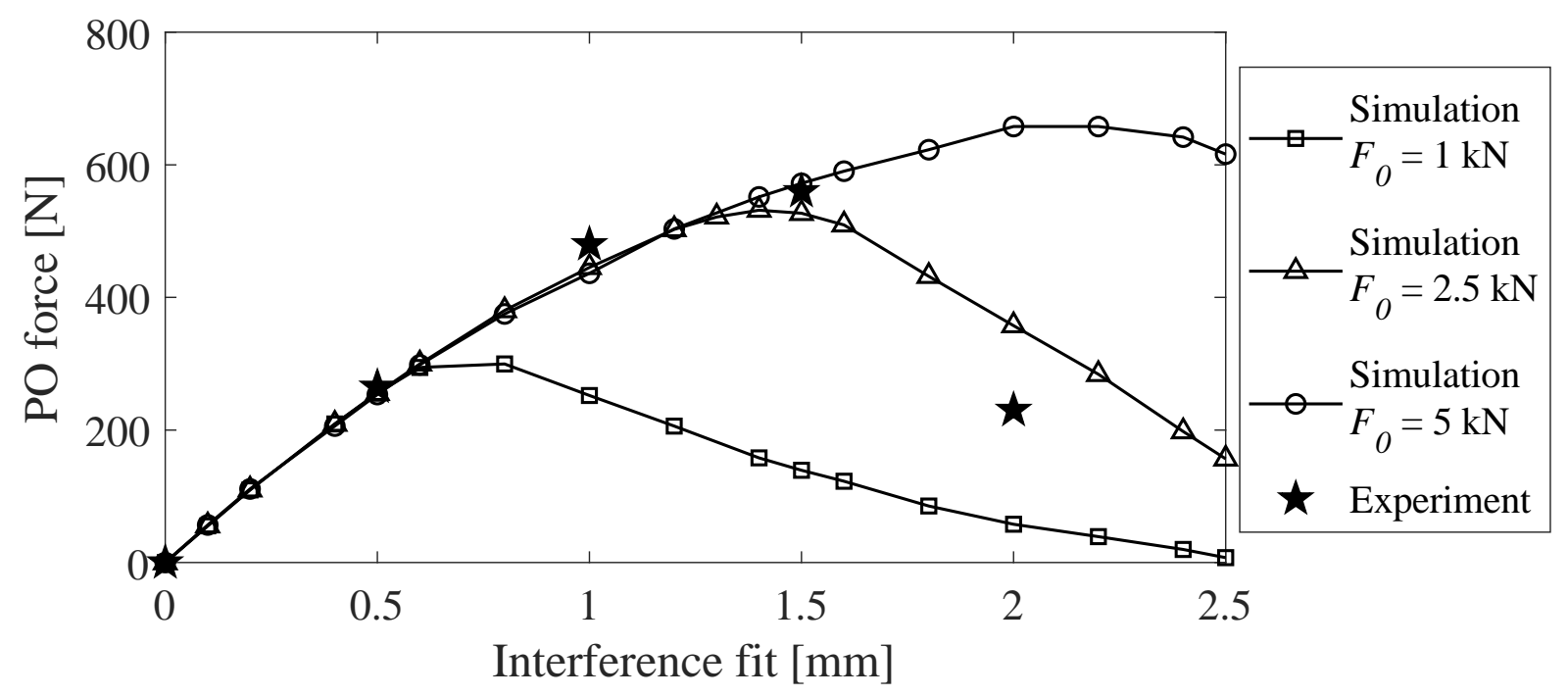


Figure 3. Variation of the pull-out force as a function of the interference fit. The experimental data is indicated with stars. The numerical results are calculated for different values of the press-fitting force with $\mu=0.3$ and $E_{b}$ $=0.2 \mathrm{GPa}$.

\section{Results}

\subsection{Reference configuration}

Figure 4 shows the variation of the vertical reaction force $R_{a}(t)$ at the upper surface of the ancillary obtained using FE simulations. Three regimes associated to the three phases of controlled displacement may be clearly identified. At the beginning of insertion phase, $R_{a}(t)=0$ because no contact occurs between the implant and bone tissue. As soon as the contact is established (segment $\mathrm{AB}$ in Fig. 4), $R_{a}(t)$ decreases as a function of the penetration depth until a predetermined value $F_{0}=-2.5$ $\mathrm{kN}$. During the insertion phase, the slope of $R_{a}$ with respect to time, is first constant and then changes after around $t=0.8 \mathrm{~s}$, which will be discussed in section 4. After the holding phase (segment $\mathrm{BC}$ ) during which $R_{a}$ is constant, the AC implant is pulled-out and the reaction force $R_{a}$ increases from negative to positive values until $R_{a}(t)$ reaches a maximum value of $444.8 \mathrm{~N}$, referred to as the pull-out (PO) force, obtained for $t=1.3 \mathrm{~s}$. Then, $R_{a}(t)$ decreases until the implant is completely removed from the bone cavity after $t=1.9 \mathrm{~s}$.

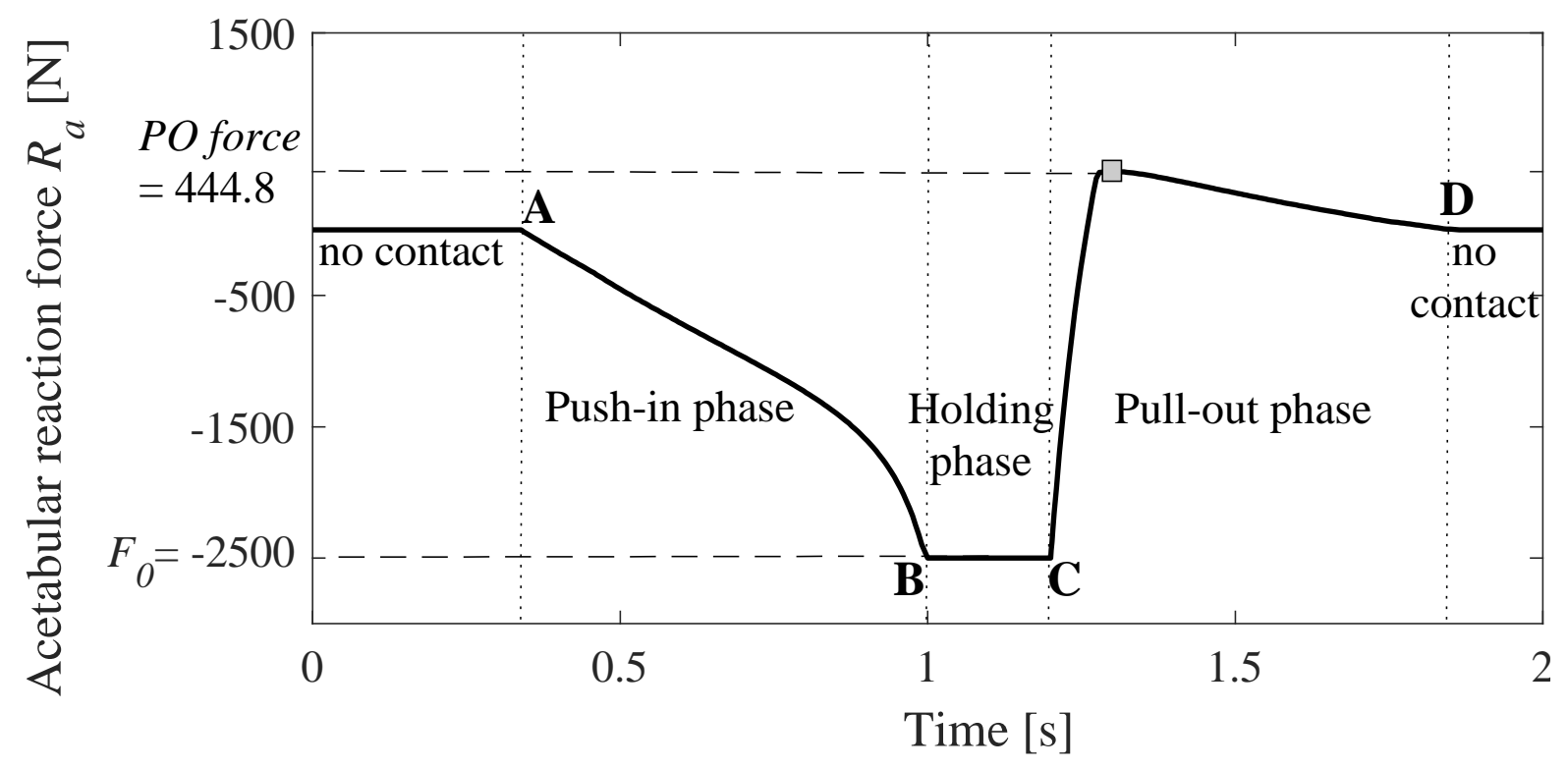

Figure 4 Variation of the reaction force $R_{a}$ as a function of time for the reference values: $\mu^{*}=0.3, E_{b}{ }^{*}=0.2 \mathrm{GPa}$ and $I F^{*}=1 \mathrm{~mm}$. The press-fitting force $F_{0}$ is taken equal to $2.5 \mathrm{kN}$.

Figure 5 shows the variation of the frictional stress $t_{T}$, the contact pressure $t_{N}$ and the polar gap $g$ (defined by the normal distance between the implant and bone surfaces) as a function of the curvilinear coordinates $r_{c}$ (which follow the internal surface of the bone cavity, see Fig. 1a) in the case of Fig. 4 for $t=1.1 \mathrm{~s}$ (when maximum insertion is achieved). In the non-contact region (i.e. for $r_{c}<r_{c}{ }^{*}$ 
$=26.8 \mathrm{~mm}), g>0$ while $t_{T}=0$ and $t_{N}=0$, as expected. The maximum value of $g$ is obtained at the bone cavity dome. In the contact region $\left(r_{c}>r_{c}{ }^{*}\right), t_{T}>0$ and $t_{N}>0$, as expected. Moreover, in the contact region, we verified (data not shown) that $\left|t_{T}\right|=\mu t_{N}$, which indicates that sliding conditions are obtained during the insertion phase until the maximal penetration depth is reached. The model predicts a maximum contact pressure $t_{N}=10.69 \mathrm{MPa}$ and a maximum frictional stress $t_{T}=2.99 \mathrm{MPa}$ near the equatorial rim, at the coordinate $r_{c}=37.35 \mathrm{~mm}$. All these results constitute a validation of the FE model.

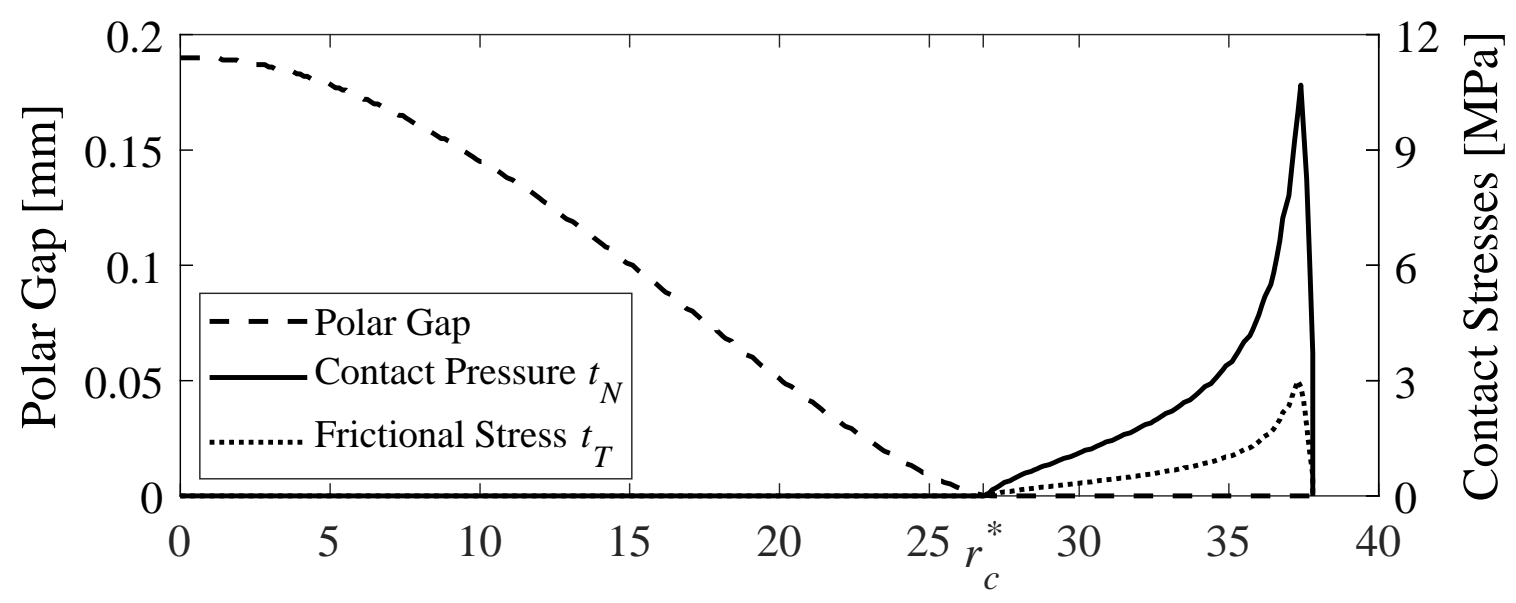

Arc Length $r_{c}[\mathrm{~mm}]$

Figure 5 Variation of the polar gap $g$, the contact pressure $t_{N}$ and the frictional stress $t_{T}$ as a function of arc length coordinate $r_{c}$ at the maximum penetration depth of the AC implant $(t=1.1 \mathrm{~s})$. The polar gap, the frictional stress and the contact pressure are calculated for the reference values: $\mu^{*}=0.3, E_{b}{ }^{*}=0.2 \mathrm{GPa}$ and $I F^{*}=1 \mathrm{~mm}$. The press-fitting force is taken equal to 2.5 $\mathrm{kN}$. The coordinate $r_{c}=r_{c}{ }^{*}=26.8 \mathrm{~mm}$ indicates the separation of the contact region with the non-contact region on the bone cavity.

\subsection{Parametric study}

\subsubsection{Effect of variations of the friction coefficient $\mu$}

Figure 6 shows that the value of the friction coefficient $\mu$ influences the time dependence of the reaction force $R_{a}$. For all values of $\mu, R_{a}(t)$ first decreases linearly at the beginning of the push-in phase, which corresponds to a constant macroscopic rigidity of the bone-implant system. For relatively low values of $\mu(\mu \leq 0.3)$, the macroscopic rigidity increases after around $\mathrm{t}=0.8 \mathrm{~s}$, while for higher values of $\mu$, the macroscopic rigidity stays constant during the entire push-in phase.

The value of the PO force is shown to depend on $\mu$. When $\mu=0, R_{a}(t)$ behaves similarly during the push-in and the pull-out phases and the PO force is equal to zero. Figure 7 shows the variation of the PO force as a function of the friction coefficient $\mu$ (for $E_{b}=E_{b}{ }^{*}$ and $I F=I F^{*}$ ). As shown in Fig. 7, the PO force is shown to increase as a function of $\mu$ for low values of $\mu$, reaches a maximum value equal to $919 \mathrm{~N}$ for $\mu=0.6$ and then slowly decreases. 


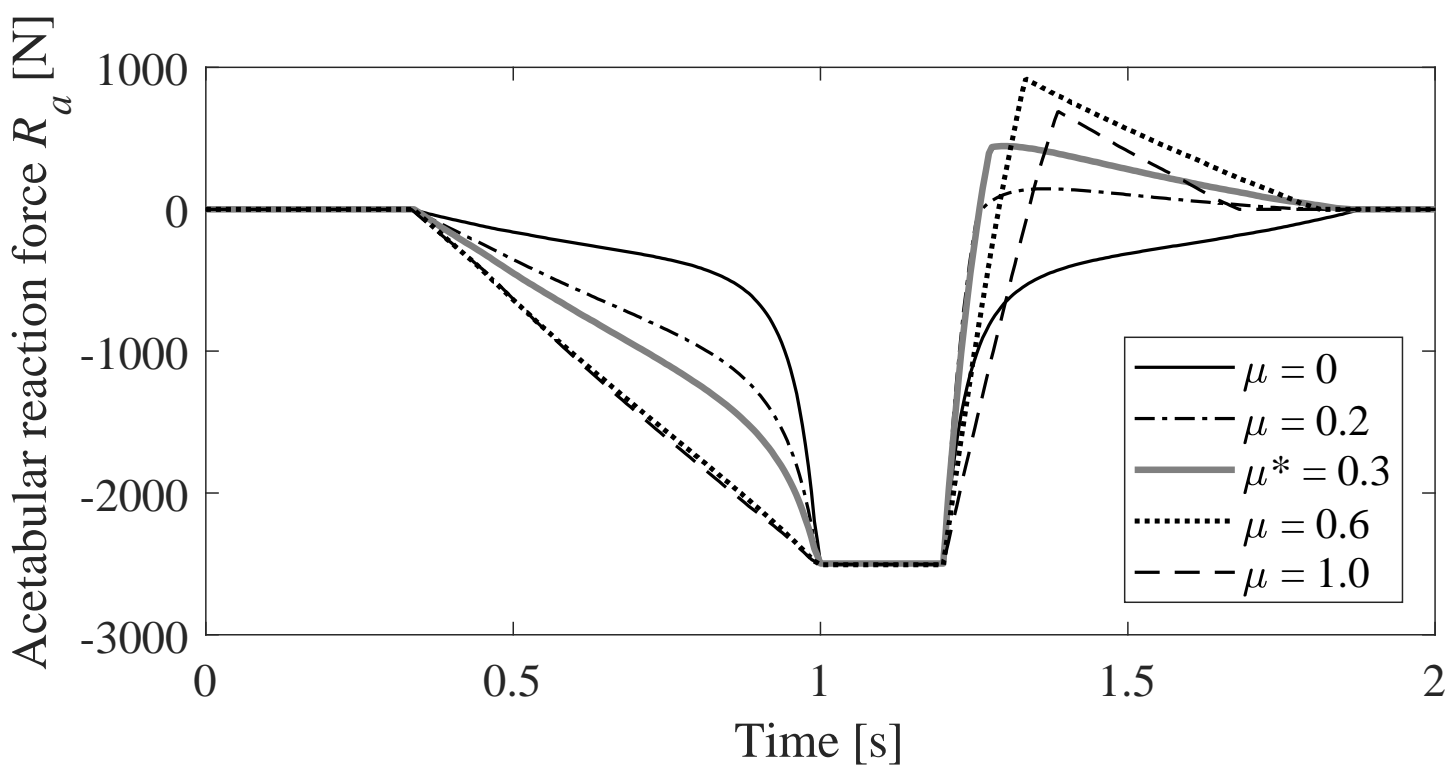

Figure 6 Variation of the reaction force $\mathrm{R}_{\mathrm{a}}$ applied to the ancillary as a function of time for different values of the friction coefficient $\mu$. The results are shown for constant values of the bone Young's modulus $E_{b}=E_{b}{ }^{*}=0.2 \mathrm{GPa}$, and the interference fit $I F=I F^{*}=1 \mathrm{~mm}$. The press-fitting force is taken equal to $2.5 \mathrm{kN}$. The curve corresponding to the reference value for $\mu=\mu^{*}=0.3$ is indicated with a solid grey line.

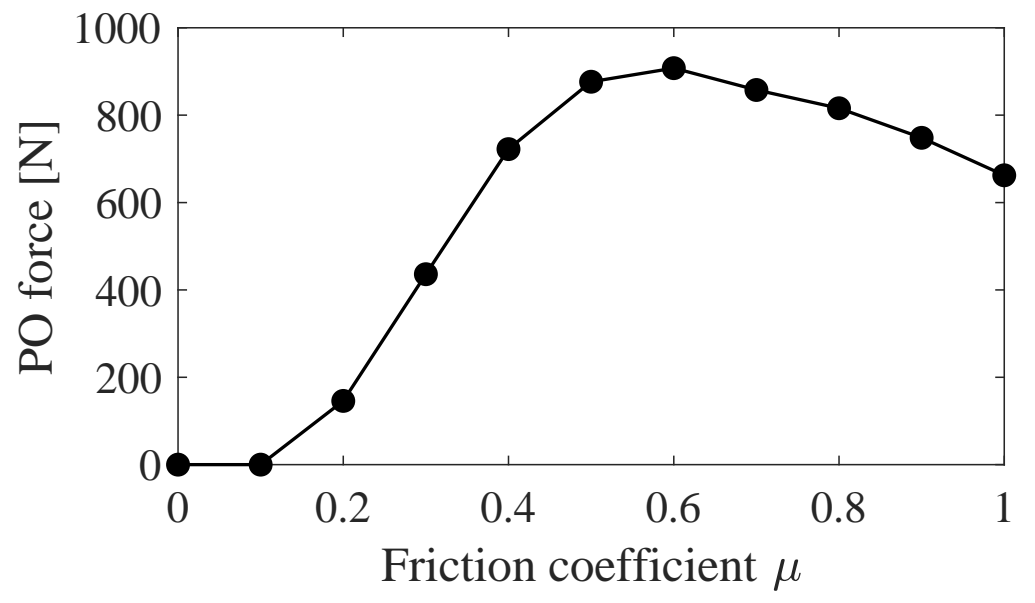

Figure 7 Variation of the pull-out (PO) force as a function of the friction coefficient $\mu$ for constant values of $E_{b}=E_{b}{ }^{*}=0.2$ $\mathrm{GPa}$ and $I F=I F^{*}=1 \mathrm{~mm}$. The press-fitting force is taken equal to $2.5 \mathrm{kN}$ for all simulations.

\subsubsection{Effect of variations of the bone Young's modulus $E_{b}$}

Figure 8 shows that for all values of $E_{b}, R_{a}$ first decreases linearly as a function of time at the beginning of the push-in phase, which corresponds to a constant macroscopic rigidity of the boneimplant system. For relatively low values of $E_{b}\left(E_{b} \leq 0.2 \mathrm{GPa}\right)$, the macroscopic rigidity increases after around $\mathrm{t}=0.8 \mathrm{~s}$, while for higher values of $E_{b}$, the macroscopic rigidity stays constant during the entire push-in phase. 


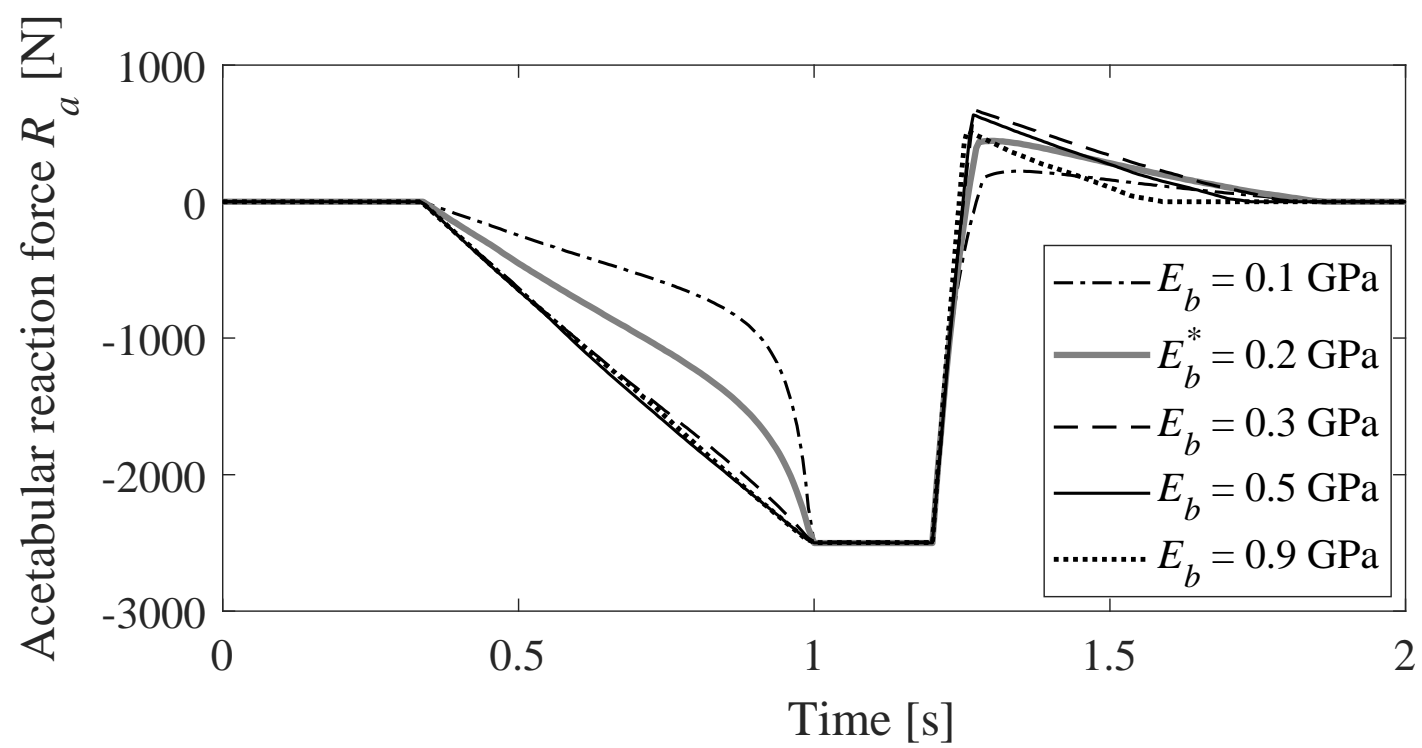

Figure 8 Variation of the reaction force $\mathrm{R}_{\mathrm{a}}$ applied to the ancillary as a function of time for different values of trabecular bone Young's modulus $E_{b}$ and for constant values of the friction coefficient $\mu=\mu^{*}=0.3$ and of the interference fit $I F=I F^{*}=$ $1 \mathrm{~mm}$. The press-fitting force is taken equal to $2.5 \mathrm{kN}$. The curve corresponding to the reference value $E_{b}{ }^{*}$ is indicated with a solid grey line.

The value of the PO force is shown to depend on $E_{b}$. Figure 9 shows that the PO force increases as a function of $E_{b}$ for low values of $E_{b}$ and then reaches a maximum value of $667 \mathrm{~N}$ for $E_{b}=0.35$ GPa. The PO force then slowly decreases for $E_{b}>0.35 \mathrm{GPa}$.

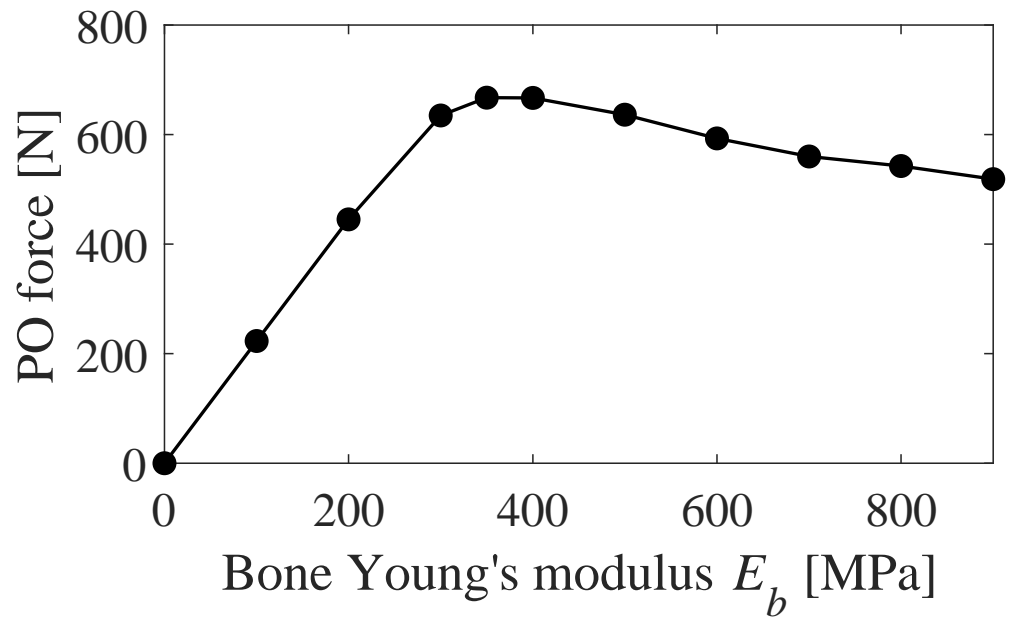

Figure 9 Variation of the pull-out force as a function of the bone Young's modulus $E_{b}$ for constant values of $\mu=\mu^{*}=0.3$ and $I F=I F^{*}=1 \mathrm{~mm}$. The press-fitting force is taken equal to $2.5 \mathrm{kN}$ for all simulations. 


\subsubsection{Effect of variations of the interference fit IF}

Figure 10 shows that for all values of $I F, R_{a}(t)$ first decreases linearly as a function of time at the beginning of the push-in phase, which corresponds to a constant rigidity of the bone-implant system. For relatively low values of $I F(I F \leq 1 \mathrm{~mm})$, the rigidity increases after around $\mathrm{t}=0.8 \mathrm{~s}$, while for higher values of $I F$, the rigidity stays constant during the entire push-in phase.

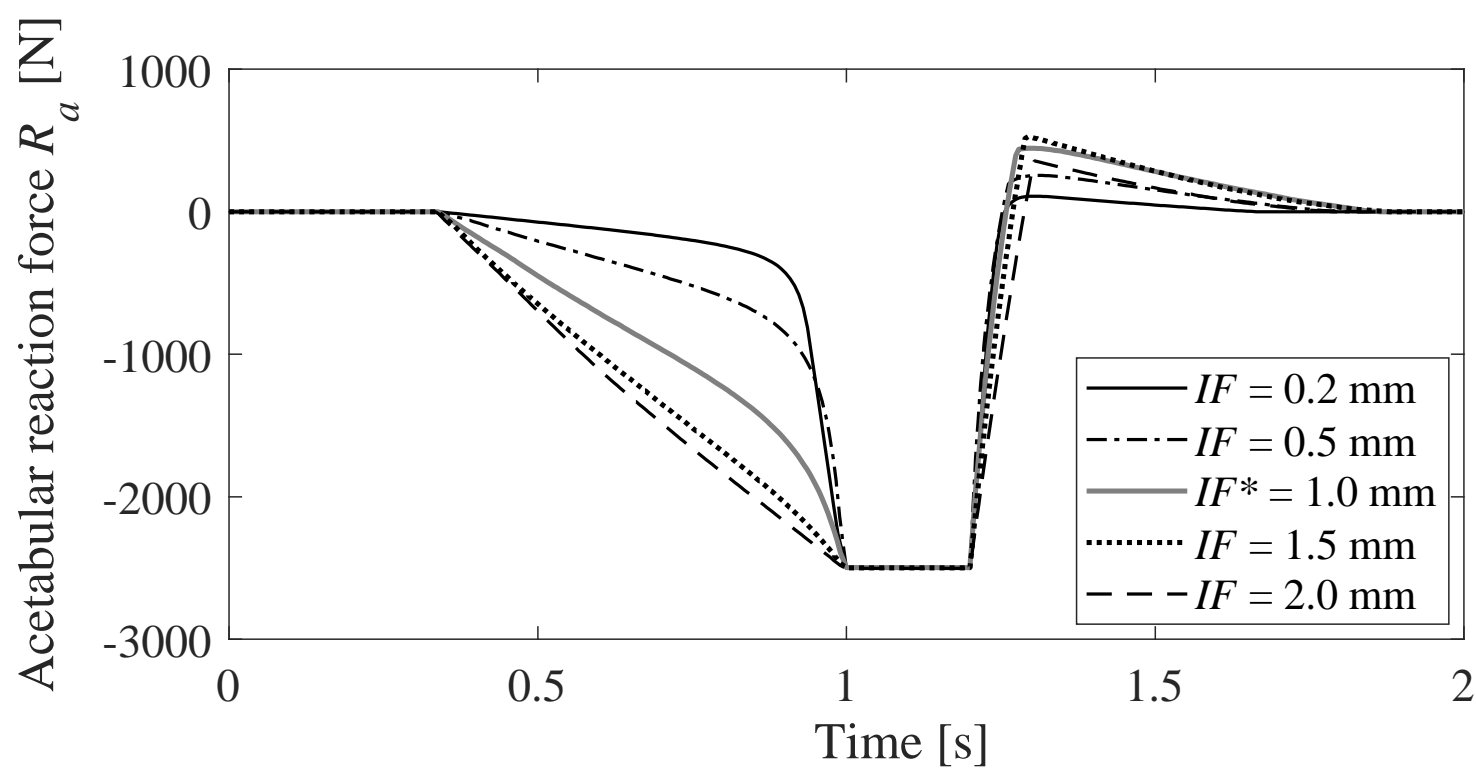

Figure 10 Variation of the reaction force $R_{a}$ applied to the ancillary as a function of time for different values of the interference fit $I F$ and for constant values of the friction coefficient $\mu=\mu^{*}=0.3$ and of the bone Young's modulus $E_{b}=E_{b}{ }^{*}=$ $0.2 \mathrm{GPa}$. The press-fitting force is taken equal to $2.5 \mathrm{kN}$. The curve corresponding to the reference value $I F^{*}$ is indicated with a solid grey line.

The value of the PO force is shown to depend on $I F$. Figure 11 shows that the PO force increases as a function of $I F$ for low values and then reaches a maximum value $P_{0}=532 \mathrm{~N}$ (referred to as optimal $P O$ force in what follows) for a given interference fit $I F_{0}=1.4 \mathrm{~mm}$ (denoted optimal interference fit in what follows). The couple $\left(I F_{0}, P_{0}\right)$ corresponds to the optimal stability condition for the reference values $E_{b}{ }^{*}$ and $\mu^{*}$. The optimal stability condition is an important information from a clinical perspective because it corresponds to the interference fit that should be obtained by the surgeon in order to maximize the implant stability. 


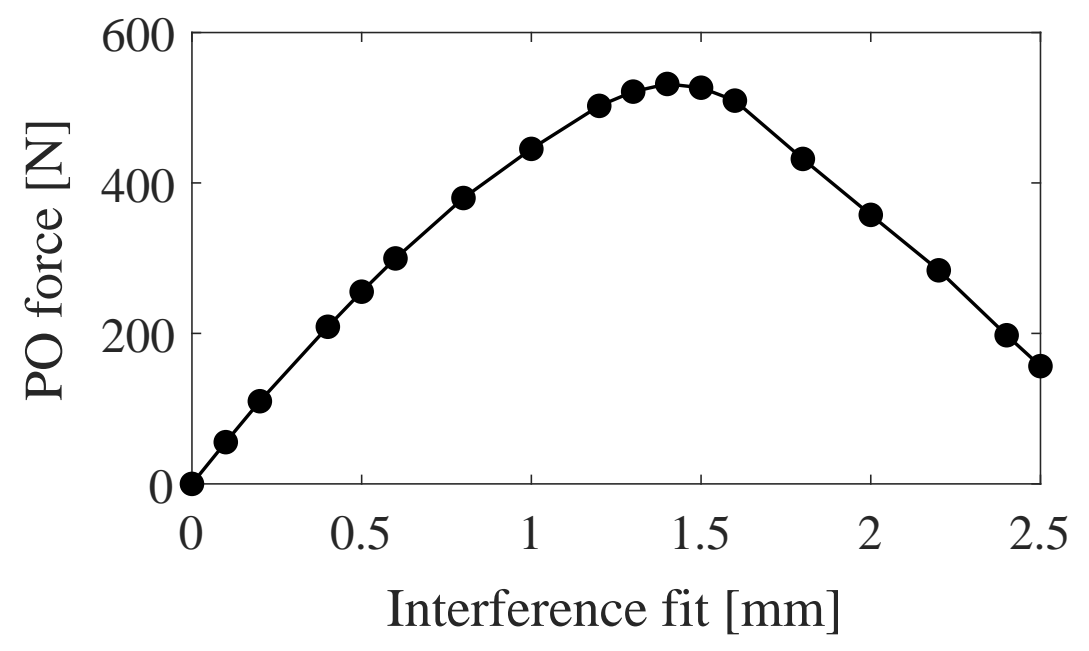

Figure 11 Variation of the pull-out force as a function of the interference fit $I F$ for constant values of $\mu=\mu^{*}=0.3$ and $E_{b}=$ $E_{b}{ }^{*}=0.2 \mathrm{GPa}$. The press-fitting force is taken equal to $2.5 \mathrm{kN}$ for all simulations.

\subsubsection{Effect of combined variations of the three parameters $I F, \mu$ and $E b$}

Figure 12 shows that a nonlinear dependence of the PO force as a function of $I F$, like results shown in Fig. 11, is obtained for all investigated values of $\mu$. The results show that the optimal pull-out force $P_{0}$ increases as a function of $\mu$. Moreover, the optimal interference fit $I F_{0}$ first increases as a function of $\mu$, for $\mu \in\{0.1 ; 0.25\}$, and then decreases for $\mu>0.25$.

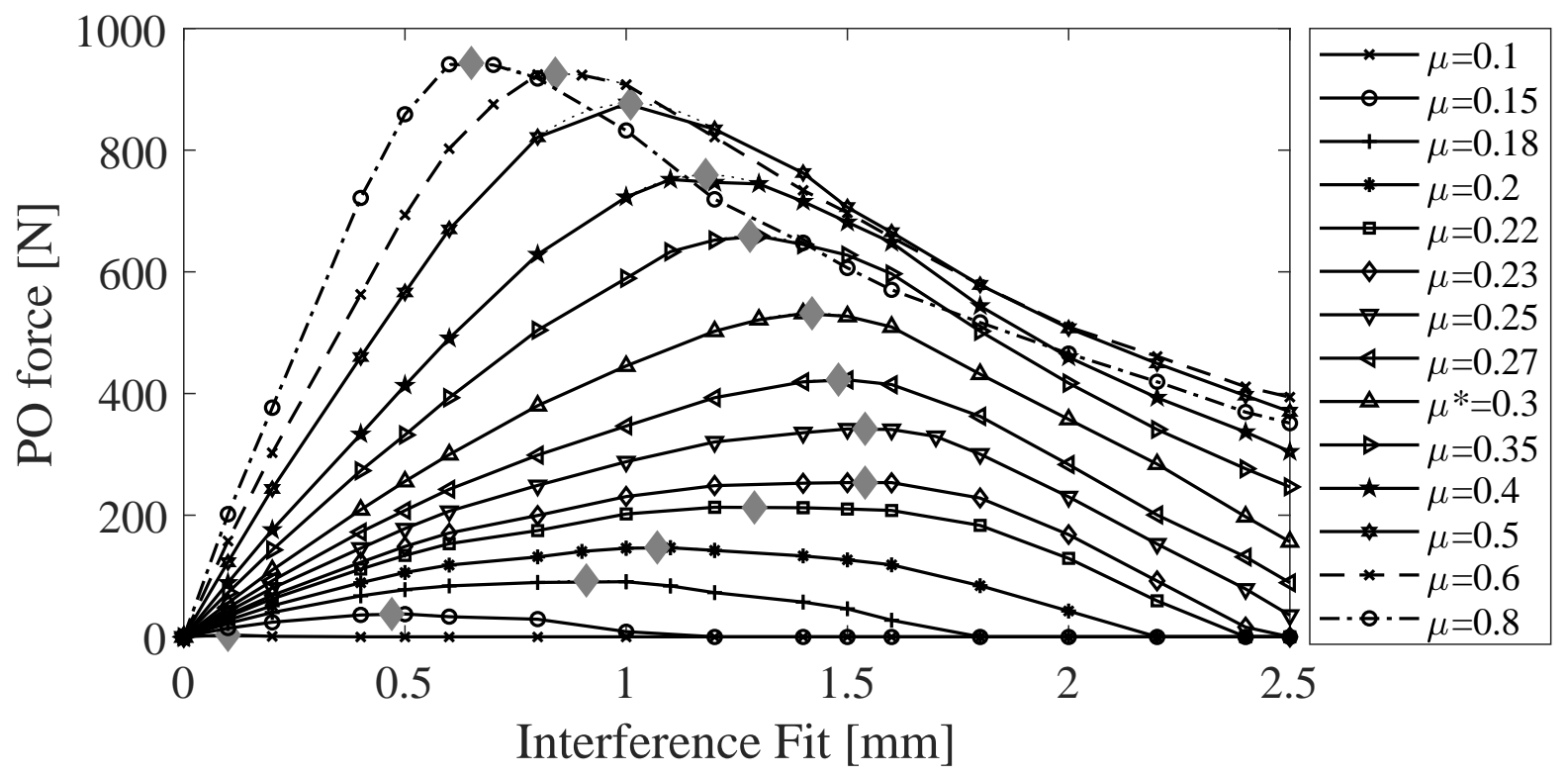

Figure 12 Variation of the pull-out force as a function of the interference fit $I F$ for several values of friction coefficient $\mu$. The value of the bone Young's modulus $E_{b}$ is taken equal to the reference value $E_{b}{ }^{*}=0.2 \mathrm{GPa}$. The value of the press-fitting force is taken equal to $2.5 \mathrm{kN}$. The optimal values of interference fit and the corresponding maximal pull-out forces $\left(I F_{0}, P_{0}\right)$ are indicated by grey diamonds.

Figure 13 shows that for three different values of $E_{b}, I F_{0}$ first increases as a function of $\mu$ until it reaches a maximum, then slowly decreases. The initial slope does not depend on $E_{b}$; however, the maximum value of $I F_{0}$ decreases as a function of $E_{b}$ and it is reached for lower values of $\mu$ as $E_{b}$ 
increases. Therefore, for $\mu>0.2$, a smaller interference fit should be considered when $E_{b}$ increases in order to obtain optimal primary stability conditions.

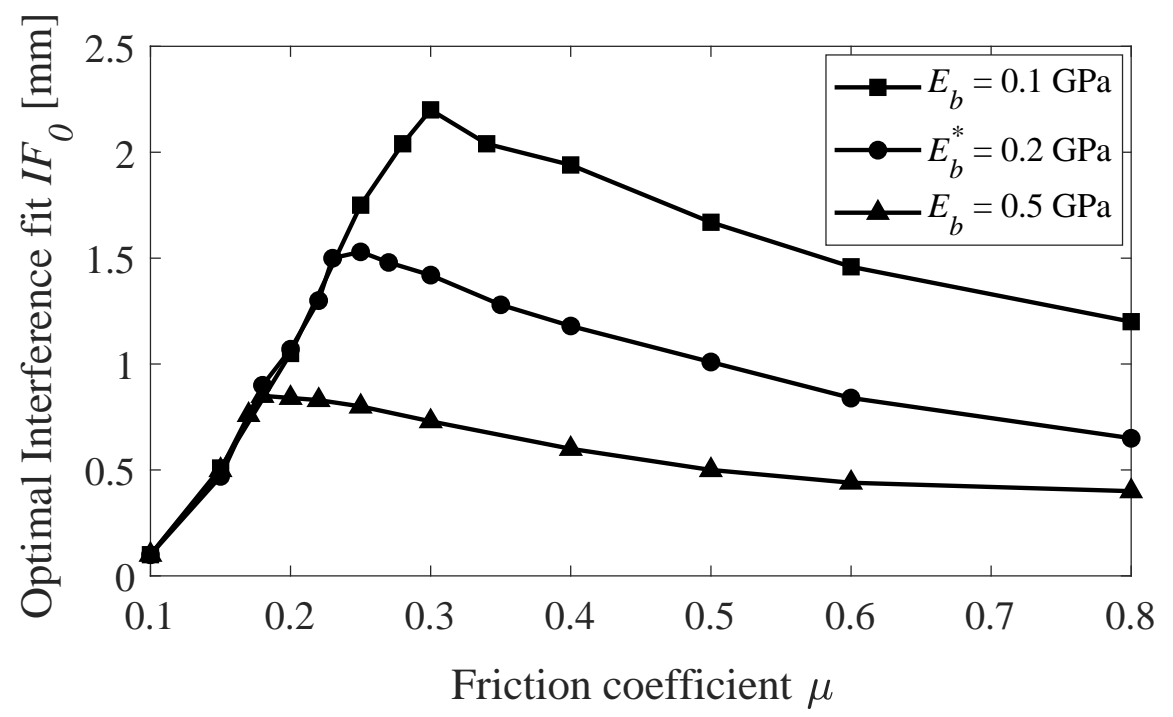

Figure 13. Variation of the optimal interference fit $I F_{0}$ as a function of the friction coefficient $\mu$ for several values of the bone Young's modulus $E_{b}$. The value of the press-fitting force is taken equal to $2.5 \mathrm{kN}$ for all simulations.

Figure 14 shows that the optimal PO force $P_{0}$ increase for $\mu$ lower than around 0.5 and then reaches a maximum. Therefore, for all values of $E_{b}$, increasing $\mu$ higher than around 0.5 does not improve the primary stability if the optimal interference fit is considered by the surgeon. However, $P_{0}$ decreases when $E_{b}$ decreases for relatively high values of $\mu$.

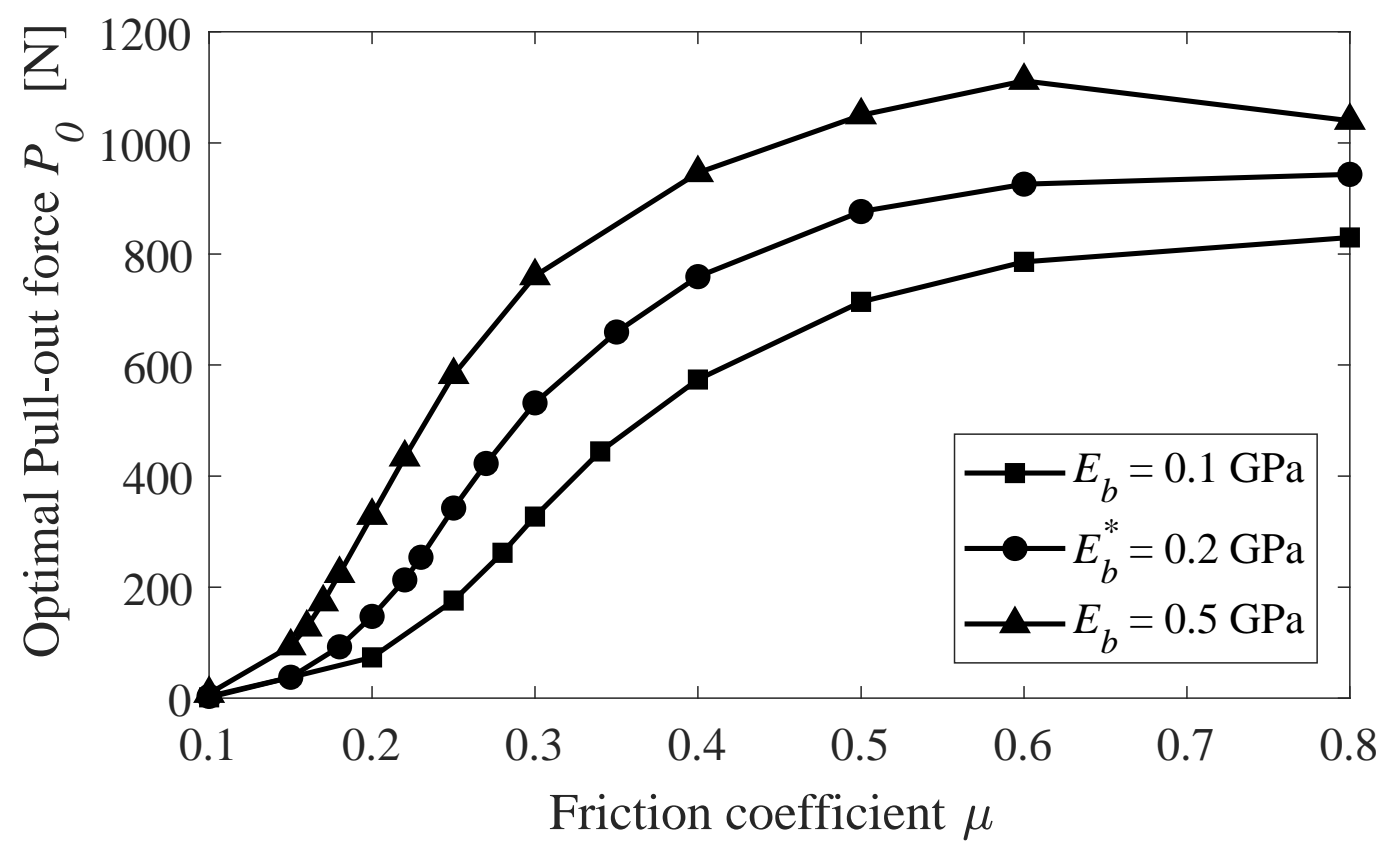

Figure 14 Variation of the optimal pull-out force $P_{0}$ as a function of the friction coefficient $\mu$ for several values of the bone Young's modulus $E_{b}$. The value of the press-fitting force is taken equal to $2.5 \mathrm{kN}$ for all simulations. 


\section{Discussion}

The present study aims to provide more insight on the interaction between an AC implant and bone tissue, leading to a better understanding of the biomechanical determinants of the AC implant primary stability, which is an important parameter for the surgical success (35). The AC implant primary stability depends on the bone mechanical properties $\left(E_{b}\right)$, on the friction coefficient $(\mu)$ and on the interference fit $(I F)$. The influence of these three parameters was investigated within their respective physiological range, using FEM-based analyses. Moreover, the mechanical behavior of the model was confirmed experimentally (see Fig. 3).

An originality of the present numerical study lies in the extensive parametrical investigation of the effect on the pull-out force of combined variations of the apparent Young's modulus of bone tissue $\left(E_{b}\right.$ $\in\{0.1-0.9\} \mathrm{GPa})$, the interference fit $(I F \in\{0.1-2.5\} \mathrm{mm})$ and the friction coefficient $(\mu \in\{0.1-1\})$. One novelty is to propose an evaluation of optimal primary stability conditions depending on $I F, \mu$ and $E_{b}$. The optimal interference fit value $I F_{0}$, which maximizes the pull-out force, is found to be related to $\mu$ (see Fig. 12) and to $E_{b}$ (see Fig. 13). The strong correlation between the different parameters may therefore require particular attention of the implant manufacturers and of the surgeons in order to maximize the AC implant primary stability.

\subsection{Comparison with the literature}

\subsubsection{Pull-out force}

As shown in Fig. 7, the PO force is found to increase as a function of $\mu$ for $\mu<0.6$. Spears, Morlock (32) found a pull-out force comprised between 234 and $798 \mathrm{~N}$ (with an insertion load increasing from 1 to $4 \mathrm{kN}$ ) for $I F=1 \mathrm{~mm}$ and $\mu=0.3$, which agrees with the results obtained herein. In the present study, a press-fitting force of $2.5 \mathrm{kN}$ was applied and the PO force was equal to $445.4 \mathrm{~N}$ (see Fig. 7) for $I F=1 \mathrm{~mm}$ and $\mu=0.3$. However, Spears (32) found a decreasing variation of the pull-out force as a function of $\mu$ for $\mu \in\{0.1-0.5\}$. Such a variation between the pull-out force and the friction coefficient may be difficult to understand because it could lead to obtain a maximal value of the PO force when $\mu$ $=0$.

The values of the PO forces obtained in the present numerical study are also consistent with the experimental results obtained by Le Cann, Galland (13), where the authors found values of the PO force comprised between $296.2 \pm 53.6 \mathrm{~N}$ and $539 \pm 19.5 \mathrm{~N}$, using bovine bone samples with an insertion load comprised between 1.8 and $4 \mathrm{kN}$ and an interference fit of $1 \mathrm{~mm}$. Although the results obtained by Le Cann, Galland (13) are useful to qualitatively validate the present model, the lack of information on the friction coefficient at the BII and on the bone mechanical properties did not allow a more accurate comparison. 


\subsubsection{Optimal stability conditions}

As shown in Fig. 13, the optimal value of the interference fit $I F_{0}$ depends on $\mu$ and on $E_{b}$, which constitutes an originality of the present work. The results emphasize the importance of the frictional behavior of the BII; which is not straightforward to determine as it depends on many factors such as implant surface treatment and bone quality (43). Previous numerical results confirm our findings concerning the existence of an optimal primary stability condition linking press-fitting and friction phenomena. Namely, Le Cann, Galland (13) implemented a 3-D model of a Sawbone block $\left(E_{b}=\right.$ $0.284 \mathrm{GPa}, v=0.3$ ) with a central hemispheric cavity in which a rigid hemispheric cup was inserted and then pulled-out within a quasi-static framework in a displacement-controlled mode. A friction coefficient $\mu=0.25$ was considered and three values of interference fit were taken into account: $0.5,1$ and $1.5 \mathrm{~mm}$. They found that the best implant stability was obtained for $I F=1 \mathrm{~mm}$, which agrees with our results $\left(I F_{0}=1-1.6 \mathrm{~mm}\right.$ for $\left.E_{b}=0.2 \mathrm{GPa}\right)$. Other authors $(32,33)$ found using numerical approaches in 2-D and 3-D framework that the best stability condition was obtained for a friction coefficient $\mu=0.2$ and an interference fit of $1 \mathrm{~mm}$.

Some authors carried out experimental pull-out test on AC implants (40, 41) and gave some indications about an optimal stability condition in terms of interference fit. Namely, Kwong, O'Connor (40) proposed the value $I F=1 \mathrm{~mm}$ as the optimal one; other authors (41) suggested a larger interference fit, $I F=2-3 \mathrm{~mm}$. Nevertheless, the above cited studies did not provide any quantitative indications about the quality of bone tissue and frictional properties of the contacting surfaces.

It remains difficult to accurately compare the present numerical results with experimental data because of the lack of quantitative information on the friction coefficient and of the difficulty of precisely machining a perfectly hemispherical cavity into bone tissue. Note that the interest of a numerical approach over an experimental one is that it provides the possibility of considering independent variations of each aforementioned parameter, which is difficult to achieve experimentally.

\subsubsection{Polar gap}

The present work focuses on the dependence of the PO force on the biomechanical environment of the AC implant. Although the PO force is an indicator of the AC implant stability (32), other factors such as for example the polar gap also influence the surgical outcome (44). The polar gap should be minimized in order to improve osseointegration quality, so that bone formation may occur in an appropriate manner (9). Spears, Morlock (32) found values for the polar gap comprised between 0.3 and $2 \mathrm{~mm}$ at the end of the insertion process for $I F=1 \mathrm{~mm}$, while Yew, Jin (34) found values for the polar gap comprised between 0.2 and $0.3 \mathrm{~mm}$ under the same conditions. These results agree with the present study (see Fig. 5), where a maximum polar gap of $0.2 \mathrm{~mm}$ is found for $\mu=0.3, I F=1 \mathrm{~mm}$ and $E_{b}=0.2 \mathrm{GPa}$ (reference set of parameters). However, Spears, Morlock (32) created a 2-D axisymmetric model of the superior region of a human pelvis in which a $1 \mathrm{~mm}$-thick layer of cortical 
bone (Young's modulus $=15.6 \mathrm{GPa}$ ) was considered at the bone cavity rim. The presence of cortical bone may lead to a slightly different biomechanical situation (see section 4.3).

\subsubsection{Contact stresses and Bone-Implant Contact}

As shown in Fig. 5, the maximal value of the contact stresses in the reference configuration is found to be localized at the bone-cavity-equatorial rim. A similar behavior is also found for all tested numerical configurations (data not shown). These results are in good agreement with previous works by several authors $(5,6,13,32,44)$, which established that the contact between the AC implant and the surrounding bone tissue mostly occurs around the equatorial rim. As shown in Fig. 5, the maximum value of the contact pressure during the full-insertion phase for the reference configuration is found to be equal to around $11 \mathrm{MPa}$. This result is in good agreement with the results obtained by Udofia, Liu (45) using a 3-D FE model where the contact pressure (averaged over the peri-equatorial zone of the acetabulum for $I F=1 \mathrm{~mm}$ ) was found to be equal to $15 \mathrm{MPa}$.

Spears, Morlock (32) determined the bone-implant contact ratio (BIC) for an interference fit equal to 1 $\mathrm{mm}$ and a press-fitting load of $4 \mathrm{kN}$. They showed that the BIC decreases from $50 \%$ for $\mu=0.2$ to 38 $\%$ for $\mu=0.4$. In this study, we found that the BIC is equal to $70 \%$ for a friction coefficient $\mu=0.3$ (see Fig. 5). The BIC values estimated in the present study are slightly higher compared to those obtained by Spears, which may be explained by the presence of the cortical bone in their model.

\subsubsection{Comparison with experimental results}

The experimental data shown in Fig. 3 agree with the numerical behavior described in subsection 3.2.3. Note that a similar behavior of the pull-out force as a function of the interference fit was also obtained in $(23,24)$. The differences between the numerical and experimental results may be explained by various experimental errors such as for example: i) the degradation of the mechanical properties (in terms of stiffness and friction) at the BII due to the multiple testing configurations, ii) the measurement of the pull-out force and iii) possible errors in the cavity reaming. Despite all these uncertainties, in the case of trabecular bone in contact with porous-coated metal, the values of $E_{b}=0.2$ GPa and $\mu=0.3$ found using the empirical approach described above are in good agreement with typical values obtained in the literature $(14,31,32,36,42)$.

\subsection{Physical interpretation of the results}

\subsubsection{Push-in phase}

The results shown in Figs. 6, 8 and 10 indicate that two regimes may be distinguished during the pushin phase. First, the macroscopic rigidity (noted $M R$ in what follows) of the bone-implant system, which is given by the slope of the force as a function of the displacement $U_{z}$ stays constant as a function of time. Note that this first regime may last during the entire push-in phase, in particular for 
relatively important values of $\mu, E_{b}$ and $I F\left(\mu>0.3, E_{b}>0.2 \mathrm{GPa}\right.$ and $\left.I F>1 \mathrm{~mm}\right)$. During this first regime, the contact between bone and the implant is restricted to the equatorial rim and occurs in sliding mode, which corresponds to the results shown in Fig. 5. The constant MR may be explained by the fact that the macroscopic configuration does not evolve as a function of time and the reaction force $R_{a}$ is transferred to bone tissue through the equatorial rim only. During this first regime, $M R$ was found to increase as a function of $\mu, E_{b}$ and $I F$. Note that this result cannot be deduced directly from Figs. 6 , 8 and 10 which show the variation of $R_{a}$ as a function of time, while the penetration $d_{0}$ obtained at time $t=1 \mathrm{~s}$ is not the same for all configurations (see subsection 2.1.2). The increase of the $M R$ as a function of $\mu$ may be explained as follow. The contact conditions at the BII correspond to a sliding mode occurring at the equatorial rim where the normal $\boldsymbol{n}$ of the BII is approximately perpendicular to the $z$ axis. Therefore, an increase of $\mu$ leads to an increase of $R_{a}$ which is given by the tangential stress $t_{T}$ at the BII. Similarly, the increase of $M R$ as a function of $I F$ (respectively $E_{b}$ ) may be explained by the fact that the normal stress $t_{N}$ at the BII increases as a function of $I F$ (respectively $E_{b}$ ), which leads to a higher value of $R_{a}$ due to the Coulomb's law (sliding occurs at the BII).

In a second regime, the $M R$ increases as a function of time during the push-in phase after a certain time which depends on the configuration, in particular in the case of Fig. 6 (respectively Fig. 8 and 10) for $\mu \leq 0.3$ (respectively $E_{b} \leq 0.2 \mathrm{GPa}$ and $I F \leq 1 \mathrm{~mm}$ ). The increase of $M R$ as a function of time may be explained by the fact that the contact area progressively increases since the bottom of the AC implant starts being in contact with bone tissue.

\subsubsection{Dependence of the pull-out force}

The nonlinear variation of the PO force as a function of $\mu$ shown in Fig. 7 may be explained as follows. For relatively low values of $\mu$, increasing $\mu$ leads to an increase of the tangential stresses at the equatorial rim, which in turn leads to an increase of the PO forces. However, when $\mu$ becomes relatively important (around 0.6), it becomes difficult to insert the AC implant into the bone cavity due to the important friction during the push-in phase, which explains the decrease of PO force as a function of the $\mu$. Thus, these two competing phenomena explain the optimal conditions of implant stability obtained for a given value of $\mu$.

Similar phenomenological explanations may be used to understand the results obtained in Figs. 9 and 11. Namely, for relatively low values of $I F$ (respectively $E_{b}$ ), increasing $I F$ (respectively $E_{b}$ ) leads to an increase of the normal stress $t_{N}$ in the equatorial rim, which in turn leads to an increase of the PO forces as a function of $I F$ (respectively $E_{b}$ ). However, when $I F$ (respectively $E_{b}$ ) becomes too high, it starts being difficult to insert the AC implant into the bone cavity, which explains the decrease of PO force as a function of the $I F$ (respectively $E_{b}$ ). Therefore, these two competing phenomena explain the optimal conditions of implant stability obtained for a given value of $I F$ (respectively $E_{b}$ ). 
The PO force has important clinical implications because it corresponds to an estimation of the AC implant primary stability. Consequently, the PO force should be maximized in clinical practice as far as possible. One of the difficulties of this problem is that the different parameters influencing the implant stability $\left(\mu, E_{b}\right.$ and $\left.I F\right)$ are determined by different stakeholders since $\mu$ depends on the implant manufacturer and on the patient, $E_{b}$ depends on the patient bone quality and $I F$ depends on the surgeon. The results obtained in Figs. 12, 13 and 14 may be used to help the surgeons and implant manufacturers optimize AC implant primary stability. In particular, the surgeon should optimize the $I F$ given the fact that $\mu$ and $E_{b}$ are fixed and are determined by the patient and the implant manufacturer. The results shown in Fig. 13 show that for relatively low values of the friction coefficient $(\mu<0.2)$, the optimal value of the $I F$ increases as a function of $\mu$ independently on the bone elastic modulus $E_{b}$. However, for relatively low values of the friction coefficient $(\mu<0.2)$, surgeons should take into account $E_{b}$ as well as $\mu$ to determine the optimal value of $I F$. In particular, for a given value of $\mu, I F$ should be lower when $E_{b}$ increases in order to optimize AC implant primary stability.

From the point of view of the implant manufacturers, the results shown in Fig. 14 indicate that increasing the friction coefficient $\mu$ higher than 0.5 is not useful because it does not lead to an increase of the optimal PO force $P_{0}$.

\subsection{Limitations}

This study has several limitations. First, a 2-D axisymmetric approximation is considered, while reaming of the bone cavity may lead to irregular shapes such as for example elliptical shapes, which may be due to the bone deformation (35). Note that obtaining a perfectly hemi-spherical bone cavity is difficult to achieve in the clinic. The precise determination of the optimal interference fit value is a complex problem and we therefore considered an idealized and standardized configuration.

Second, bone material properties were homogeneous, elastic and isotropic because this assumption allows to assess the influence of a small number of parameters in a controlled configuration. Trabecular bone in the acetabulum is known to be heterogeneous and anisotropic because of the remodeling phenomena related to the complex mechanical stress field occurring around the implant. Note that the effect of the bone anisotropy on the primary stability of AC implants has been investigated in a previous paper by our group (4). Furthermore, the chosen reference value for the bone Young's modulus $E_{b}{ }^{*}=0.2 \mathrm{GPa}$ is in agreement with a recent experimental and numerical campaign carried out by Henys and Capek (37). The behavior of bone tissue during the insertion of an AC implant is likely to be elastoplastic, which was not taken into account in this study. However, a recent study (46) recommends considering plasticity models for numerical simulations when a deformation of the bone tissue beyond the yield strength is likely to occur, which is not the case in our study. Although some studies $(5,13)$ took into account elastoplastic effects, the influence of plasticity on the macroscopic behavior of the AC implant remains unclear. 
Third, a quasi-static configuration was considered and all dynamic aspects were neglected, similarly as what was done in $(13,32,33)$. Note that a previous study (20) by our group precisely focused on the insertion process of an AC implant by considering a dynamic modeling. Considering a dynamic modeling is important to model the insertion that is realized with hammer impacts. However, using dynamic modeling would not modify the pull-out test, which is the main subject of the present study.

Fourth, the cortical bone layer near the cavity rim was not considered in order to model the experimental configuration, to simplify the geometrical configuration and to study the influence of a limited number of parameters. Note that the cortical layer may also be removed during the surgical reaming procedure.

Fifth, the proposed model should be validated experimentally. However, this is a difficult task due to all the approximation described above and to the fact that it remains difficult to measure with precision the actual friction coefficient, interference fit and bone elastic modulus (bone being heterogeneous, viscoelastic and anisotropic). Instead, we simply provide a comparison between the numerical and analytical results. Moreover, a design of experiments (DOE) approach (47) would have been useful to investigate the influence of the various parameters on the PO force and to create a surrogate model of the bone-implant system in terms of primary stability. In the present study, we made the simplest choice of an OFAT (one-factor-at-time) design to perform a preliminary screening, which allowed to identify the existence of an "optimal stability condition" linking the interference fit and the friction coefficient. Then, the study of the interaction between the interference fit, friction coefficient and bone Young's modulus was made only with reference to the optimal stability condition because this condition is of clinical relevance and should clearly be pointed out. Future studies should consider a full DOE analysis.

\section{Conclusions}

The present study investigates the dependence of the AC implant primary stability on its environment including the interference fit, the friction coefficient and bone properties. This study emphasizes the existence of an optimal primary stability condition, which depends in a coupled manner on the friction coefficient, on the bone properties and on the interference fit. The numerical results are found to be consistent with experimental measurements as well as with previous experimental and numerical studies. These results may be useful to improve clinical practice (such as for example, the drilling procedure) or the $\mathrm{AC}$ implant conception by providing a better understanding of the phenomena occurring at the BII. However, the proposed in silico model needs to be improved in order to better match the clinical conditions. Accordingly, the real configuration of the human hemi-pelvis together with the anisotropy of the trabecular bone must be considered. To this aim, a 3-D FEM-based investigation will be carried-out. 


\section{Acknowledgements}

This project has received funding from the European Research Council (ERC) under the European Union's Horizon 2020 research and innovation program (grant agreement No 682001, project ERC Consolidator Grant 2015 BoneImplant).

Conflict of Interest: the authors declare that they have no conflict of interest.

\section{References}

1. Sun H, Inaoka H, Fukuoka Y, Masuda T, Ishida A, Morita S. Range of motion measurement of an artificial hip joint using CT images. Med Biol Eng Comput. 2007;45(12):1229-35.

2. Hsu JT, Lai KA, Chen Q, Zobitz ME, Huang HL, An KN, et al. The relation between micromotion and screw fixation in acetabular cup. Comput Methods Programs Biomed. 2006;84(1):34-41.

3. Toossi N, Adeli B, Timperley AJ, Haddad FS, Maltenfort M, Parvizi J. Acetabular components in total hip arthroplasty: is there evidence that cementless fixation is better? J Bone Joint Surg Am. 2013;95(2):168-74.

4. Nguyen V-H, Rosi G, Naili S, Michel A, Raffa M-L, Bosc R, et al. Influence of anisotropic bone properties on the biomechanical behavior of the acetabular cup implant: a multiscale finite element study. Computer Methods in Biomechanics and Biomedical Engineering. 2017;20(12):131225 .

5. Janssen D, Zwartele RE, Doets HC, Verdonschot N. Computational assessment of press-fit acetabular implant fixation: the effect of implant design, interference fit, bone quality, and frictional properties. Proc Inst Mech Eng H. 2010;224(1):67-75.

6. Hothi HS, Busfield JJ, Shelton JC. Explicit finite element modelling of the impaction of metal press-fit acetabular components. Proc Inst Mech Eng H. 2011;225(3):303-14.

7. Sotto-Maior BS, Rocha EP, de Almeida EO, Freitas-Junior AC, Anchieta RB, Del Bel Cury AA. Influence of high insertion torque on implant placement: an anisotropic bone stress analysis. Braz Dent J. 2010;21(6):508-14.

8. Mathieu V, Vayron R, Richard G, Lambert G, Naili S, Meningaud JP, et al. Biomechanical determinants of the stability of dental implants: influence of the bone-implant interface properties. $\mathbf{J}$ Biomech. 2014;47(1):3-13.

9. Haiat G, Wang HL, Brunski J. Effects of biomechanical properties of the bone-implant interface on dental implant stability: from in silico approaches to the patient's mouth. Annu Rev Biomed Eng. 2014;16:187-213.

10. Branemark PI, Hansson BO, Adell R, Breine U, Lindstrom J, Hallen O, et al. Osseointegrated implants in the treatment of the edentulous jaw. Experience from a 10-year period. Scand J Plast Reconstr Surg Suppl. 1977;16:1-132.

11. Albrektsson T, Branemark PI, Hansson HA, Lindstrom J. Osseointegrated titanium implants. Requirements for ensuring a long-lasting, direct bone-to-implant anchorage in man. Acta Orthop Scand. 1981;52(2):155-70.

12. Mukherjee K, Gupta S. Combined Bone Ingrowth and Remodeling Around Uncemented Acetabular Component: A Multiscale Mechanobiology-Based Finite Element Analysis. Journal of Biomechanical Engineering. 2017;139(9):091007--12.

13. Le Cann S, Galland A, Rosa B, Le Corroller T, Pithioux M, Argenson JN, et al. Does surface roughness influence the primary stability of acetabular cups? A numerical and experimental biomechanical evaluation. Med Eng Phys. 2014;36(9):1185-90. 
14. Damm NB, Morlock MM, Bishop NE. Friction coefficient and effective interference at the implant-bone interface. Journal of Biomechanics. 2015;48(12):3517-21.

15. Goldman AH, Armstrong LC, Owen JR, Wayne JS, Jiranek WA. Does Increased Coefficient of Friction of Highly Porous Metal Increase Initial Stability at the Acetabular Interface? J Arthroplasty. 2016;31(3):721-6.

16. Díaz-Pérez F, García-Nieto E, Ros A, Claramunt R. Best estimation of spectrum profiles for diagnosing femoral prostheses loosening. Medical Engineering \& Physics. 2014;36(2):233-8.

17. Rieger JS, Jaeger S, Schuld C, Kretzer JP, Bitsch RG. A vibrational technique for diagnosing loosened total hip endoprostheses: an experimental sawbone study. Med Eng Phys. 2013;35(3):32937.

18. Georgiou AP, Cunningham JL. Accurate diagnosis of hip prosthesis loosening using a vibrational technique. Clin Biomech (Bristol, Avon). 2001;16(4):315-23.

19. Goossens Q, Leuridan S, Henyš P, Roosen J, Pastrav L, Mulier M, et al. Development of an acoustic measurement protocol to monitor acetabular implant fixation in cementless total hip Arthroplasty: A preliminary study. Medical Engineering \& Physics. 2017.

20. Michel A, Nguyen V, Bosc R, Vayron R, Hernigou P, Naili S, et al. Finite element model of the impaction of a press-fitted acetabular cup. Med Biol Eng Comput. 2017;17:781-91.

21. Mathieu V, Michel A, Flouzat Lachaniette CH, Poignard A, Hernigou P, Allain J, et al. Variation of the impact duration during the in vitro insertion of acetabular cup implants. Med Eng Phys. 2013; 35(11):1558-63.

22. Michel A, Bosc R, Mathieu V, Hernigou P, Haiat G. Monitoring the press-fit insertion of an acetabular cup by impact measurements: influence of bone abrasion. Proc Inst Mech Eng $\mathrm{H}$. 2014;228(10):1027-34.

23. Michel A, Bosc R, Vayron R, Haiat G. In vitro evaluation of the acetabular cup primary stability by impact analysis. J Biomech Eng. 2015;137(3).

24. Michel A, Bosc R, Sailhan F, Vayron R, Haiat G. Ex vivo estimation of cementless acetabular cup stability using an impact hammer. Med Eng Phys. 2016;38(2):80-6.

25. Michel A, Bosc R, Meningaud JP, Hernigou P, Haiat G. Assessing the Acetabular Cup Implant Primary Stability by Impact Analyses: A Cadaveric Study. PLoS One. 2016;11(11):e0166778.

26. Tijou A, Rosi G, Hernigou P, Flouzat-Lachaniette CH, Haiat G. Ex Vivo Evaluation of Cementless Acetabular Cup Stability Using Impact Analyses with a Hammer Instrumented with Strain Sensors. Sensors (Basel). 2017;18(1).

27. Hsu JT, Chang CH, Huang HL, Zobitz ME, Chen WP, Lai KA, et al. The number of screws, bone quality, and friction coefficient affect acetabular cup stability. Med Eng Phys. 2007;29(10):108995.

28. Phillips AT, Pankaj, Usmani AS, Howie CR. The effect of acetabular cup size on the shortterm stability of revision hip arthroplasty: a finite element investigation. Proc Inst Mech Eng $\mathrm{H}$. 2004;218(4):239-49.

29. Ma W, Zhang X, Wang J, Zhang Q, Chen W, Zhang Y. Optimized design for a novel acetabular component with three wings. A study of finite element analysis. Journal of Surgical Research. 2013;179(1):78-86.

30. Goebel P, Kluess D, Wieding J, Souffrant R, Heyer H, Sander M, et al. The influence of head diameter and wall thickness on deformations of metallic acetabular press-fit cups and UHMWPE liners: a finite element analysis. J Orthop Sci. 2013;18(2):264-70.

31. Dammak M, Shirazi-Adl A, Schwartz M, Jr., Gustavson L. Friction properties at the bonemetal interface: comparison of four different porous metal surfaces. J Biomed Mater Res. 1997;35(3):329-36.

32. Spears IR, Morlock MM, Pfleiderer M, Schneider E, Hille E. The influence of friction and interference on the seating of a hemispherical press-fit cup: a finite element investigation. J Biomech. 1999;32(11):1183-9.

33. Spears IR, Pfleiderer M, Schneider E, Hille E, Morlock MM. The effect of interfacial parameters on cup-bone relative micromotions. A finite element investigation. $\mathrm{J}$ Biomech. 2001;34(1):113-20.

34. Yew A, Jin ZM, Donn A, Morlock MM, Isaac G. Deformation of press-fitted metallic resurfacing cups. Part 2: Finite element simulation. Proc Inst Mech Eng H. 2006;220(2):311-9. 
35. Taylor M, Prendergast PJ. Four decades of finite element analysis of orthopaedic devices: Where are we now and what are the opportunities? Journal of Biomechanics. 2015;48(5):767-78.

36. Dalstra M, Huiskes R, Odgaard A, Van Erning L. Mechanical and textural properties of pelvic trabecular bone. Journal of biomechanics. 1993;26(4-5):523-35.

37. Henys P, Capek L. Material model of pelvic bone based on modal analysis: a study on the composite bone. Biomech Model Mechanobiol. 2017;16(1):363-73.

38. Lebon F, Raous M. Multibody contact problem including friction in structure assembly. Computers \& Structures. 1992;43(5):925-34.

39. Laursen T. Computational Contact and Impact Mechanics - Fundamentals of Modeling Interfacial Phenomena in Nonlinear Finite Element Analysis. Verlag S, Editor 2003.

40. Kwong LM, O'Connor DO, Sedlacek RC, Krushell RJ, Maloney WJ, Harris WH. A quantitative in vitro assessment of fit and screw fixation on the stability of a cementless hemispherical acetabular component. J Arthroplasty. 1994;9(2):163-70.

41. Macdonald W, Carlsson LV, Charnley GJ, Jacobsson CM. Press-fit acetabular cup fixation: principles and testing. Proc Inst Mech Eng H. 1999;213(1):33-9.

42. Biemond JE, Aquarius R, Verdonschot N, Buma P. Frictional and bone ingrowth properties of engineered surface topographies produced by electron beam technology. Archives of Orthopaedic and Trauma Surgery. 2011;131(5):711-8.

43. Shirazi-Adl A, Dammak M, Paiement G. Experimental determination of friction characteristics at the trabecular bone/porous-coated metal interface in cementless implants. J Biomed Mater Res. 1993;27(2):167-75.

44. Amirouche F, Solitro G, Broviak S, Gonzalez M, Goldstein W, Barmada R. Factors influencing initial cup stability in total hip arthroplasty. Clin Biomech (Bristol, Avon). 2014;29(10):1177-85.

45. Udofia I, Liu F, Jin Z, Roberts P, Grigoris P. The initial stability and contact mechanics of a press-fit resurfacing arthroplasty of the hip. Bone \& Joint Journal. 2007;89(4):549-56.

46. Schulze, C, Vogel D, Sander M, Bader R. Calibration of crushable foam plasticity models for synthetic bone material for use in finite element analysis of acetabular cup deformation and primary stability. Computer Methods in Biomechanics and Biomedical Engineering. 2019;22(1):25-37.

47. Anderson, M J., and Whitcomb P J. DOE simplified: practical tools for effective experimentation. Productivity press, 2016. 